\title{
WSB1 overcomes oncogene-induced senescence by targeting ATM for degradation
}

Jung Jin Kim ${ }^{1, *}$, Seung Baek Lee ${ }^{1, *}$, Sang-Yeop $\mathrm{Yi}^{2}$, Sang-Ah Han ${ }^{3}$, Sun-Hyun Kim ${ }^{4}$, Jong-Min Lee, Seo-Yun Tong ${ }^{5}$, Ping Yin ${ }^{1}$, Bowen Gao ${ }^{1}$, Jun Zhang ${ }^{6}$, Zhenkun Lou ${ }^{1}$

${ }^{I}$ Department of Oncology, Mayo Clinic, Rochester, MN 55905, USA; ${ }^{2}$ Department of Medical Education, International St. Mary's Hospital, College of Medicine, Catholic Kwandong University, Incheon 404834, Republic of Korea; ${ }^{3}$ Department of Surgery, School of Medicine, Kyung Hee University, Seoul 130872, Republic of Korea; ${ }^{4}$ Department of Family Medicine, International St. Mary's Hospital, College of Medicine, Catholic Kwandong University, Incheon 404834, Republic of Korea; ${ }^{5}$ Department of Obstetrics and Gynecology, School of Medicine, Kyung Hee University, Seoul 130872, Republic of Korea; ${ }^{6}$ Department of Laboratory Medicine and Pathology, Mayo Clinic, Rochester, MN 55905, USA

Oncogene-induced senescence (OIS) or apoptosis through the DNA-damage response is an important barrier of tumorigenesis. Overcoming this barrier leads to abnormal cell proliferation, genomic instability, and cellular transformation, and finally allows cancers to develop. However, it remains unclear how the OIS barrier is overcome. Here, we show that the E3 ubiquitin ligase WD repeat and SOCS box-containing protein 1 (WSB1) plays a role in overcoming OIS. WSB1 expression in primary cells helps the bypass of OIS, leading to abnormal proliferation and cellular transformation. Mechanistically, WSB1 promotes ATM ubiquitination, resulting in ATM degradation and the escape from OIS. Furthermore, we identify CDKs as the upstream kinase of WSB1. CDK-mediated phosphorylation activates WSB1 by promoting its monomerization. In human cancer tissue and in vitro models, WSB1-induced ATM degradation is an early event during tumorigenic progression. We suggest that WSB1 is one of the key players of early oncogenic events through ATM degradation and destruction of the tumorigenesis barrier. Our work establishes an important mechanism of cancer development and progression in premalignant lesions.

Keywords: ATM; oncogene-induced senescence (OIS); tumorigenesis; WD repeat and SOCS box-containing protein 1 (WSB1)

Cell Research (2017) 27:274-293. doi:10.1038/cr.2016.148; published online 13 December 2016

\section{Introduction}

Oncogene-induced senescence (OIS) is an important cellular response for protection against cancer development [1-4]. Senescence is frequently found in premalignant lesions, but not in cancerous tissues [2,5]. Accordingly, this barrier is inactivated before cancer can develop [6]. Activation of oncogenes, such as $c-M y c$ and Ras, is a source of senescence and/or apoptosis through

*These two authors contributed equally to this work.

Correspondence: Zhenkun Lou

Tel: 1-507-284-2702; Fax: 1-507-266-4906

E-mail: lou.zhenkun@mayo.edu

Received 20 June 2016; revised 26 September 2016; accepted 29 September 2016; published online 13 December 2016 the activation of ARF, leading to p53 accumulation. Recently, evidence has emerged that the DNA-damage response (DDR) pathway is activated by oncogenic stress and triggers p53 stabilization, apoptosis, and senescence [7-9]. This response is due to collapsed replication fork, DNA replication stress, and DNA damage that may accompany hyperproliferation, leading to recruitment and activation of the serine-threonine kinase ataxiatelangiectasia mutated (ATM) [7, 10, 11]. Activated components of this DDR signaling pathway, such as pATM, pChk2, or 53BP1, are detected in various preneoplastic lesions but are lost in cancers [10, 12-14]. These findings suggest that the oncogene-induced DDR must be overcome during the tumorigenesis process. In support of this, inhibition or knockdown of ATM prevents OIS and promotes cell transformation [15-20]. 
It has also been shown that oncogene-induced DDR is essential for the induction of senescence, a barrier in preneoplastic lesions in vivo $[9,15,21]$.

ATM is a major upstream kinase of the DDR pathway $[22,23]$. Loss of ATM function is associated with both increased genomic instability and compromised p53 activation. Furthermore, several studies suggest that ATM expression and activity is lower during tumorigenesis in pre-neoplastic lesions [24-26]. However, the underlying mechanism for ATM downregulation is not clear. Understanding how ATM is regulated, especially in preneoplastic lesions, will have important implications in revealing the overcoming of OIS during tumorigenesis.

WSB1 has been reported to be an E3-ubiquitin ligase, which contains seven WD40 repeats and a SOCS box at the C-terminus $[27,28]$. The WSB1 gene is a transcriptional target of numerous onco-proteins, including c-Myc, HIF-1, and CREB-ATF [29, 30]. Several studies suggest that WSB1 expression might be positively correlated with tumor incidence and be associated with tumor development in pancreatic cancer, hepatocellular carcinoma, and salivary gland tumors [29, 31-33]. WSB1 also contributes to tumor metastasis through promoting pVHL degradation [34]. However, whether and how WSB1 contributes to tumor initiation remains unknown.

In this study, we found that WSB1 regulates ATM stability, and its overexpression results in OIS destruction and cell transformation through ATM degradation in premalignant lesions. More interestingly, WSB1 is upregulated in human pre-malignant tumors and is negatively associated with ATM expression. Therefore, our results reveal a critical function of WSB1 during tumor initiation as an E3 ligase of ATM.

\section{Results}

\section{WSB 1 promotes cell transformation and tumor initiation}

WSB 1 is overexpressed in cancer; however, its mechanism of action remains largely unclear. To examine whether WSB1 is involved in tumorigenesis, we expressed $H$-Ras ${ }^{V 12}$ in primary mouse embryonic fibroblasts (MEFs), which can induce OIS through the DDR [3, 17, 18] and INK4-ARF pathways [5, 35]. Consistent with previous publications, expressing $\mathrm{H}-\mathrm{Ras}^{\mathrm{V} 12}$ in MEFs (H-Ras ${ }^{\mathrm{V} 12}$-MEFs) caused cellular senescence, characterized by enhanced senescenceassociated $\beta$-galactosidase (SA- $\beta$-gal) staining, flattened cell morphology, and upregulation of p53, p21, and p16 (Figure 1A-1C). Interestingly, when we co-expressed $H$-Ras ${ }^{V 12}$ and $W S B 1$, cellular senescence was considerably decreased (Figure 1A and 1B). p53 and p21 expression were also decreased in cells overexpressing $H$-Ras ${ }^{V 12}$ and WSB1, but p16 was not (Figure 1C). Whereas MEFs expressing $\mathrm{H}-\mathrm{Ras}^{\mathrm{V} 12}$ alone showed reduced proliferation, MEFs expressing H-Ras ${ }^{\mathrm{V} 12}$ and WSB1 continued to proliferate (Figure 1D and Supplementary information, Figure S1A). Furthermore, expression of WSB1 with $H-\operatorname{RaS}^{V 12}$ greatly increased colony formation (Figure 1E). To confirm these results, we expressed GFP control or WSB 1-GFP together with H-Ras ${ }^{V 12}$ in IMR-90 human lung fibroblasts (Figure 1F). Consistent with $\mathrm{H}-\mathrm{Ras}^{\mathrm{V} 12}$ expressing MEFs, H-Ras ${ }^{\mathrm{V} 12}$ expression in IMR-90 induced cellular senescence, while co-expression of WSB1-GFP inhibited senescence-associated phenotypes, such as SA- $\beta$-gal staining and morphologic changes (Figure 1F). Thus, WSB1 helps overcome OIS. Next, to confirm whether WSB1 contributes to tumorigenesis in ex vivo models, we seeded $\mathrm{H}-\mathrm{Ras}^{\mathrm{V} 12}$-expressing cells or cells co-expressing H-Ras ${ }^{V 12}$ and WSB1 in a threedimensional (3-D) organoid culture system. All tumor cells are embedded in 3-D microenvironments in the body; therefore, 3-D culture systems are closer to live tissue environments than two-dimensional (2-D) culture systems and represent an important way to study untransformed and neoplastic cells [36, 37]. We found that cells co-expressing $H$-Ras ${ }^{V 12}$ and $W S B 1$, but not expressing $H$-Ras ${ }^{V 12}$ alone, grew in 3-D culture systems (Figure $1 \mathrm{G}$ and $1 \mathrm{H})$. These data suggest that WSB1 overexpression is sufficient to overcome OIS and promote cell transformation.

It is well-established that expressing both c-Myc and $\mathrm{H}-\mathrm{Ras}^{\mathrm{V} 12}$ can help overcome OIS for primary cells. We also asked whether or not WSB1 is required for this process. Interestingly, with PROMO, a transcription factor-binding site prediction program, WSB1 is predicted to be a Myc target gene. We also found that Myc expression could increase WSB 1 mRNA and protein levels (Supplementary information, Figure S1B). To test whether WSB1 is required for the escape of OIS induced by c-Myc and $\mathrm{H}-\mathrm{Ras}^{\mathrm{V} 12}$, we knocked down WSB1 in MEFs expressing both $c-M y c$ and H-Ras ${ }^{V 12}$. Cells expressing $c-M y c$ and $H-R_{a s}{ }^{V 12}$ initially showed senescence, but eventually escaped from OIS and became transformed. However, loss of WSB1 rendered cells resistant to Ras and c-Myc-induced transformation, as revealed by sustained senescence (Figure 1I). These results suggest that WSB1 is both sufficient and required for overcoming OIS.

\section{WSB1 negatively regulates ATM levels}

We next investigated how WSB1 helps to overcome OIS. DDR is a barrier of tumorigenesis and plays an important role in OIS $[14,17]$. ATM plays a critical 
A

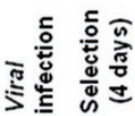
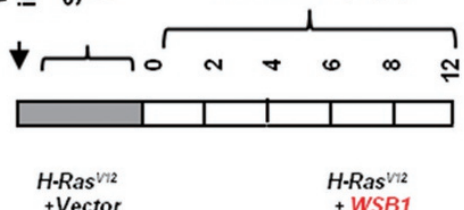

+Vecto

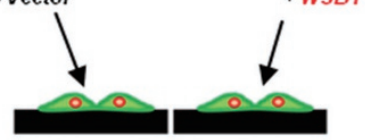

Virus infection

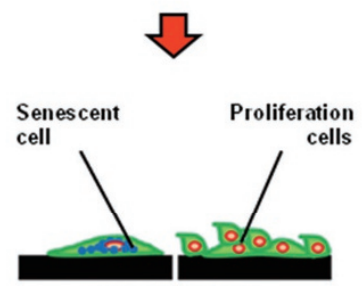

SA-p-gal staining
B

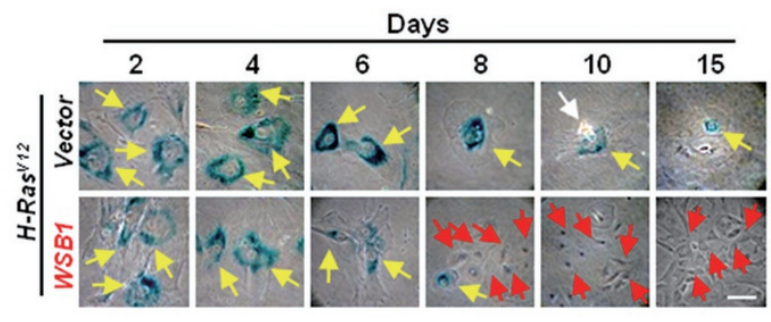

C

$\frac{H-\operatorname{Ras}^{112}}{\frac{W A}{W S B 1}}$ days

DA HA

-ー-

ロேーー- p21

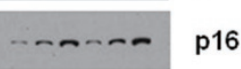

-nanana H-Ras

ananan $\beta$-actin

$\mathbf{D}$

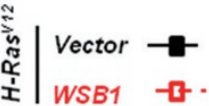

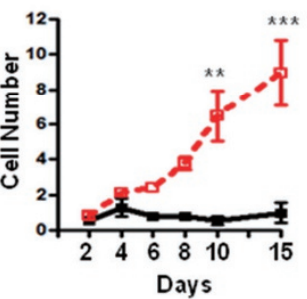

E

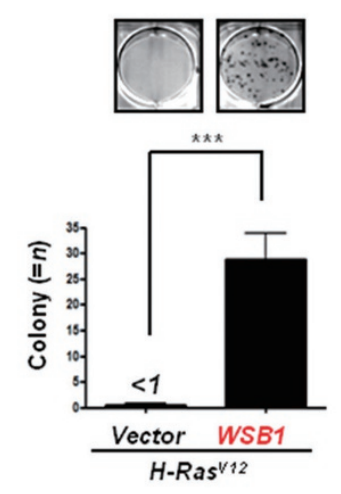

G

2D culture

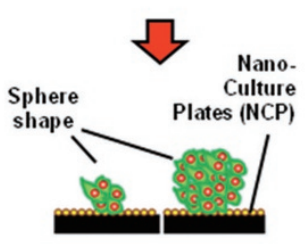

3D culture

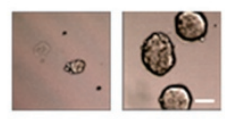

F

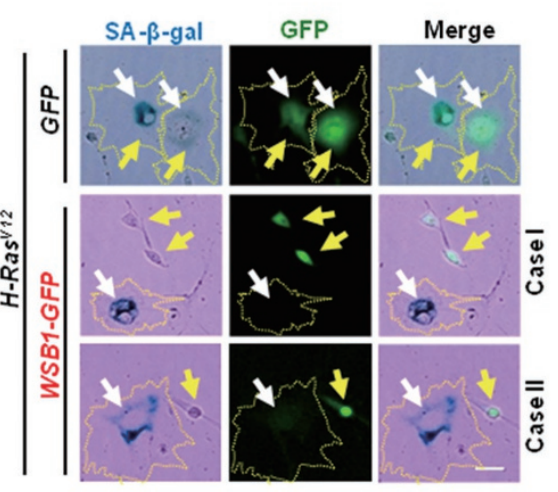

H

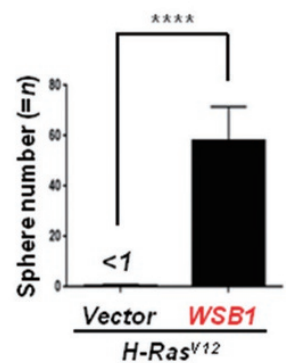

I
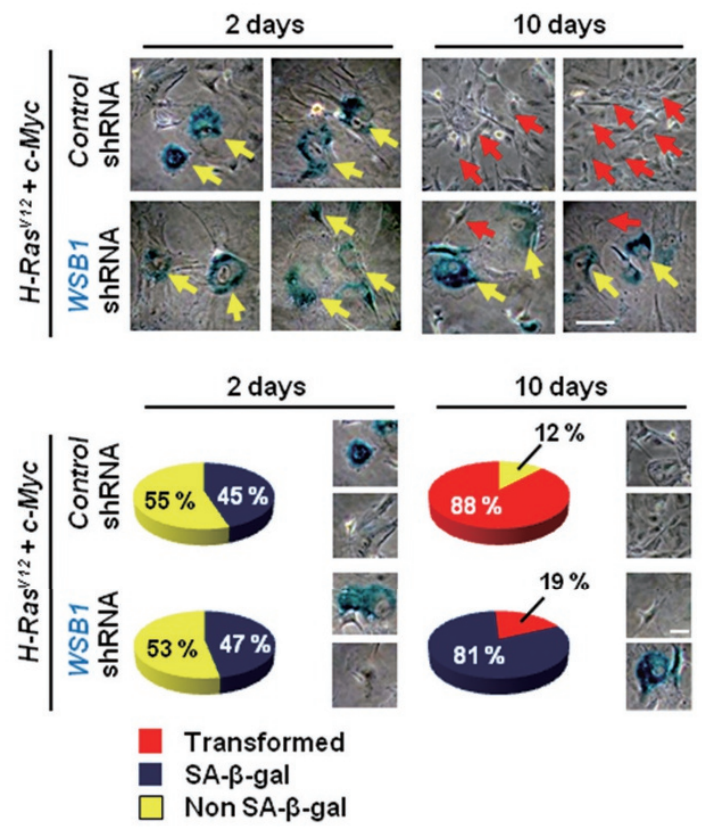

- SA- $\beta$-gal

Non SA- $\beta$-gal

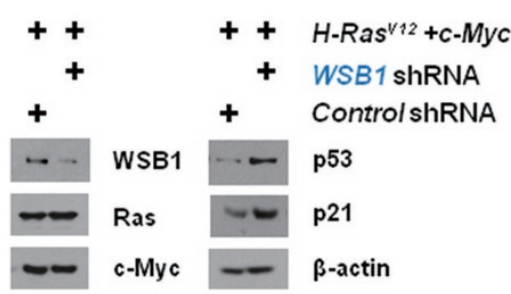


role in DDR, acting to activate p53 and suppress tumorigenesis in response to oncogene activation. OIS is bypassed in ATM-knockdown cells or deficient cells [17, $38,39]$. ATM level is also decreased in several tumors [40, 41]; however, the mechanism of ATM downregulation is not clear. Interestingly, we observed decreased ATM staining and levels in cells overexpressing WSB1 (Figure 2A-2C). These results led us to hypothesize that WSB1 might negatively regulate ATM levels and that ATM might be a major target of WSB1 in overcoming OIS.

To further test whether ATM is a major target in WSB1-mediated effects during tumor initiation, we knocked down ATM in primary MEFs and expressed $H-\operatorname{Ras}^{V 12}$ and $c-M y c$ to induce cell transformation. Colony-formation assays were performed to investigate long-term effects of WSB1 on proliferation. Both control and ATM-depleted primary MEFs showed colonyforming ability when expressing $H-R_{a s}{ }^{V 12}$ and $c-M y c$. However, WSB1 knockdown inhibited cell transformation in WT MEFs, but did not affect cell transformation in $A T M$-depleted MEFs (Figure 2D). We also obtained similar results using $A T M^{+/+}$and $A T M^{1-}$ immortal MEFs (Supplementary information, Figure S2). These results suggest that ATM is a major target of WSB1 in inducing cell transformation.

WSB1 regulates ATM levels by promoting ATM ubiquitination

WSB1 is a known E3 ligase, and several substrates, such as DIO2 (D2) [42], HIPK2 [27], and pVHL [34] have been identified. Therefore, we hypothesized that ATM is ubiquitinated by WSB1, which results in its downergulation. To test this hypothesis, we first examined the ability of WSB1 to interact with ATM. The association of endogenous ATM with WSB1 was detected via co-immunoprecipitation, and this binding was increased after MG132 treatment, which increased both ATM and WSB1 levels (Figure 3A). To identify the regions of ATM involved in binding WSB1, we performed glutathione S-transferase (GST) pull-down experiments using 8 overlapping GST-ATM fragments spanning full-length ATM [43]. WSB1 bound strongly to GST-ATM-5 (a.a. 1 690-2 120) and weakly to GSTATM-8 (a.a. 2 680-3 056, encompassing the kinase domain; Figure 3B). We further determined whether WSB 1 could regulate ATM ubiquitination. We found that WSB1 overexpression increased ATM ubiquitination (Figure 3C). In addition, in cells depleted of WSB1, ATM stability increased (Figure 3D). Furthermore, p53 stability, which is regulated by ATM, was also increased in WSB1-depleted cells. However, we did not find WSB1 to regulate ATR or DNA-PK stability, nor did it interact with ATR or regulate ATR expression (Figure 3D-3F). These results suggest that WSB1 regulates ATM levels through the ubiquitin-proteasome pathway.

As some ATM ubiquitination sites were revealed by mass spectrometry analysis (http://www.phosphosite. org), we analyzed whether these sites were ubiquitinated by WSB1. Of them, mutation of either K1323 or K2025 to arginine (R) substantially reduced ATM ubiquitination (Figure $3 \mathrm{G}$ ), suggesting that these two sites might be major sites of ubiquitination mediated by WSB1. As shown in Figure 3H, double mutation of both K1323 and K2025 residues (2KR) largely abolished ATM ubiquitination induced by WSB 1 overexpression. We therefore predicted that the $2 \mathrm{KR}$ mutant would be resistant to WSB1-mediated downregulation. To test this hypothesis, we stably expressed Flag-tagged WT ATM or ATM mutants (K1323R, K2025R, K1323R/ $\mathrm{K} 2025 \mathrm{R}$ ) in $A T M^{-1}$ immortal MEFs (Figure 3I). The

Figure 1 Oncogene-induced senescence is overcome by WSB1. (A) Schematic of the experiments. (B) Primary MEFs were infected with the indicated viruses and analyzed for senescence by SA- $\beta$-gal staining (top, examples; bottom, quantification of senescent or transformed cells). Yellow arrows indicate SA- $\beta$-gal-stained cells for senescence; red arrows indicate transformed cells; white arrow indicates a dead cell. The results represent the means $( \pm$ SE) of three independent experiments performed in triplicate. ${ }^{* * *} P<0.001$ versus control by one-way ANOVA; N.S., non-specific. Scale bar, $10 \mu \mathrm{m}$. (C-E) Primary MEFs were infected with the indicated viruses and were analyzed for senescence by immunoblotting (C), cell counting, stained as in Supplementary information, Figure S1A (D), and colony formation (E). The results represent the means $\left( \pm \mathrm{SE}\right.$ ) of three independent experiments performed in triplicate. ${ }^{* *} P<0.01$ and ${ }^{* * *} P<0.001$ versus control cells by one-way ANOVA. (F) IMR-90 cells were infected with the indicated constructs under $H$-Ras ${ }^{\mathrm{V} 12}$ activation and analyzed for senescence by SA- $\beta$-gal and immunofluorescence staining (GFP or WSB1-GFP). White arrows indicate SA- $\beta$-gal-stained cells for senescent; yellow arrows, infected cells. (G, H) Primary MEFs were infected with the indicated viruses. The cells were applied on 3-D organoid cultures system. Schematic of the experiments (G); sphere number in 3-D organoid culture (H). Scale bar, $10 \mu \mathrm{m} .{ }^{* * *} P<0.0001$ versus control cells by one-way ANOVA. (I) Primary MEFs were infected with the indicated constructs under $\mathrm{H}$-Ras ${ }^{\mathrm{V} 12}$ and $c-M y c$ activation and analyzed for senescence by SA- $\beta$-gal (top) and the quantification of senescent or transformed cells (middle). Yellow arrows indicate senescent cells by SA- $\beta$-gal staining; red arrows, transformed cells. Bar, $10 \mu \mathrm{m}$. Cell lysates were then blotted with the indicated antibodies (bottom). The results represent the means $( \pm$ $\mathrm{SE})$ of three independent experiments performed in triplicate. 
A

$$
\mid
$$

2
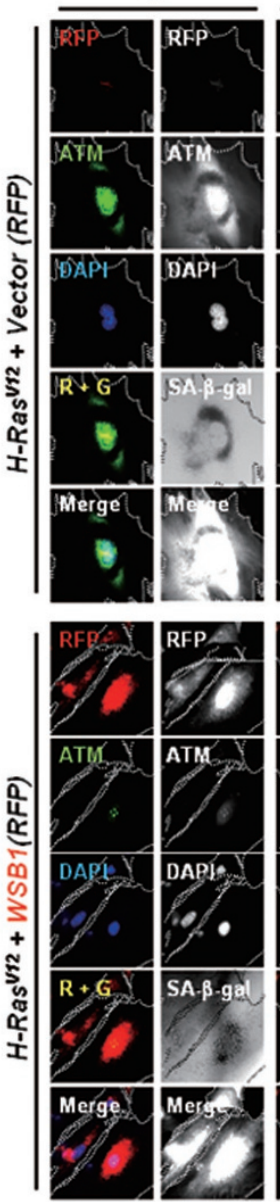

4
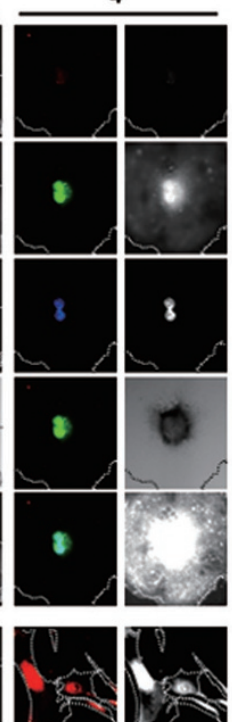
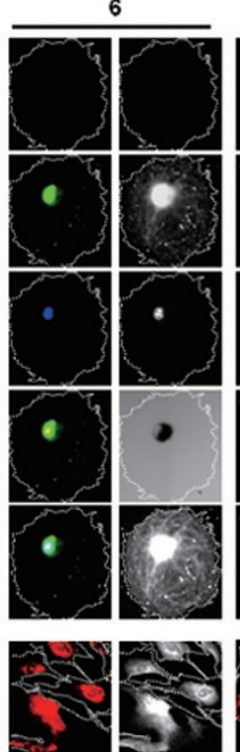

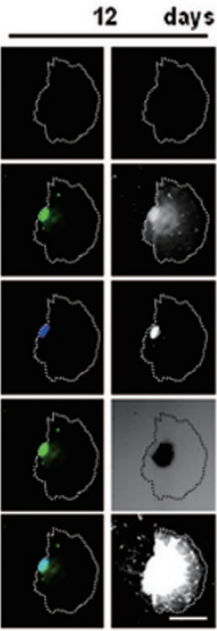

B
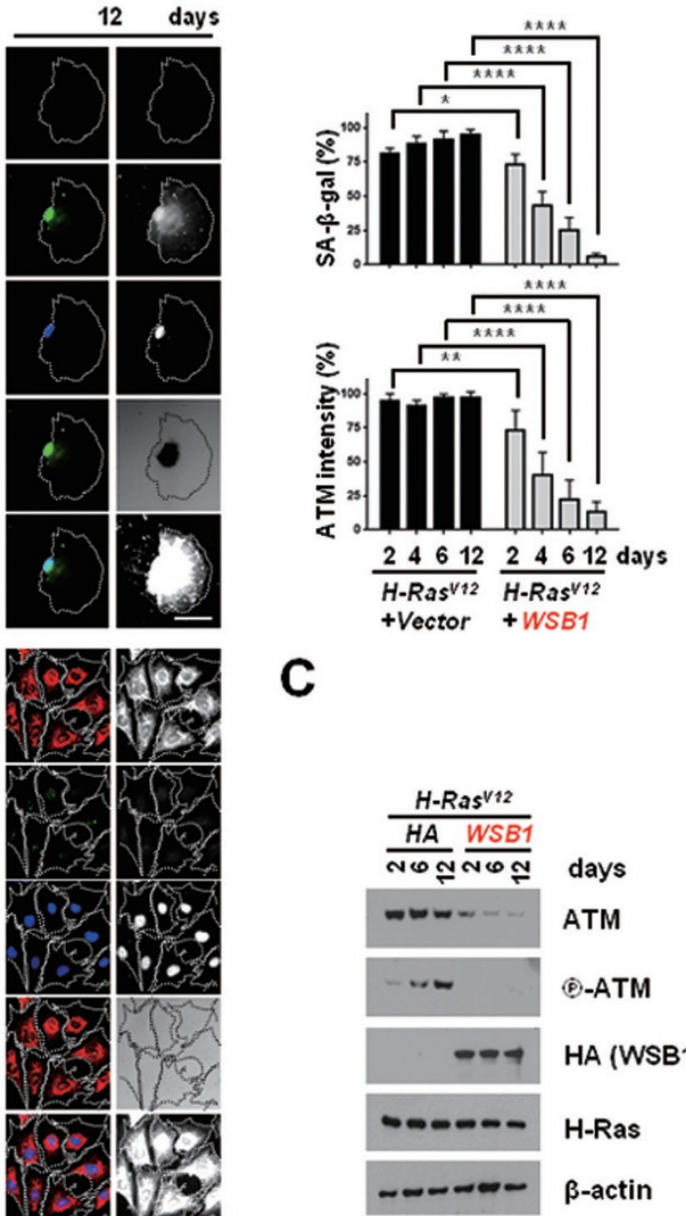

D
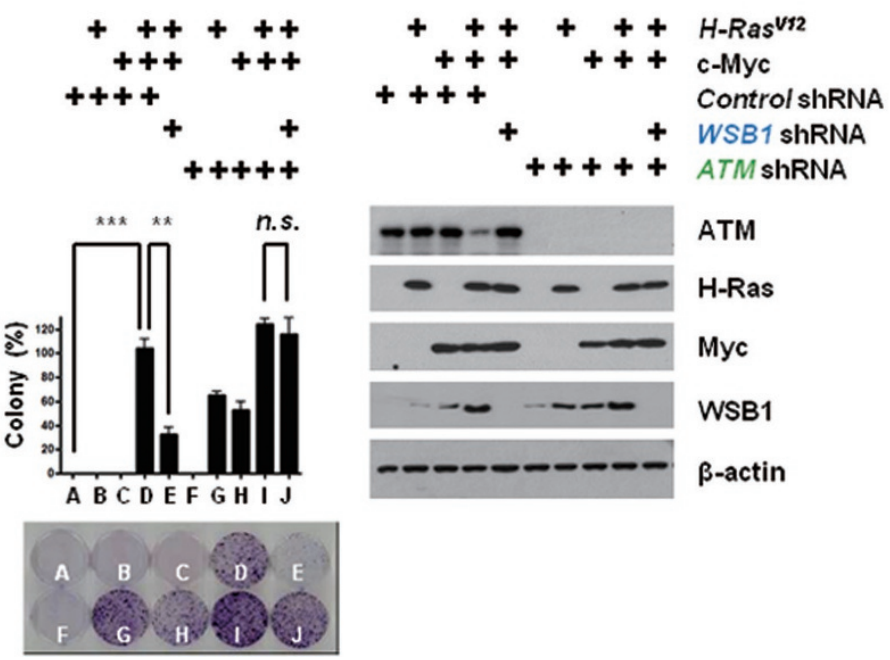

Figure 2 WSB1 negatively regulates ATM protein levels. (A-C) Primary MEFs were infected with the indicated viruses and analyzed for SA- $\beta$-gal staining and ATM expression (A). Quantification of senescent cells (top) or ATM expression (bottom, B) and immunoblot of ATM (C). Scale bar, $10 \mu \mathrm{m}$. ${ }^{*} P<0.05,{ }^{* *} P<0.01$, and ${ }^{* * * *} P<0.0001$ versus control cells by one-way ANOVA. (D) Primary MEFs were infected with the indicated viruses and analyzed for tumorigenesis by colony-formation assay (left). Cell lysates were then blotted with the indicated antibodies (right). The results represent the means ( \pm SE) of three independent experiments performed in triplicate. ${ }^{* *} P<0.01$ and ${ }^{* * *} P<0.001$ versus control cells by one-way ANOVA. 
A

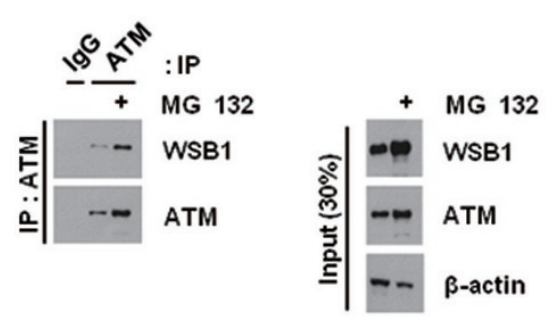

C

D
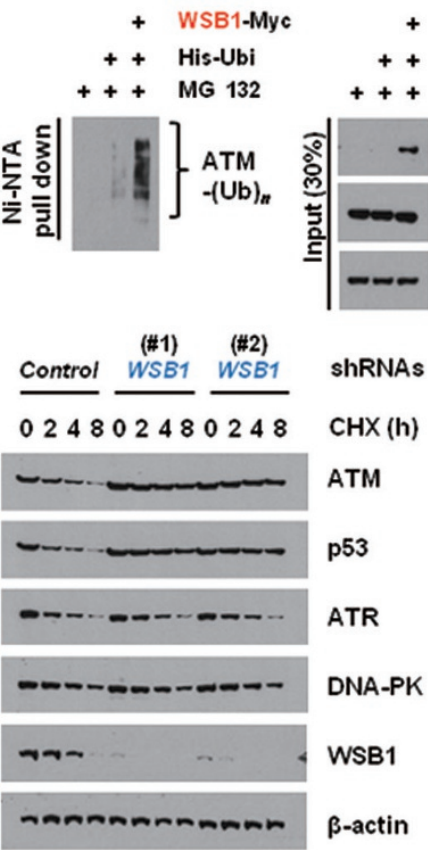

G

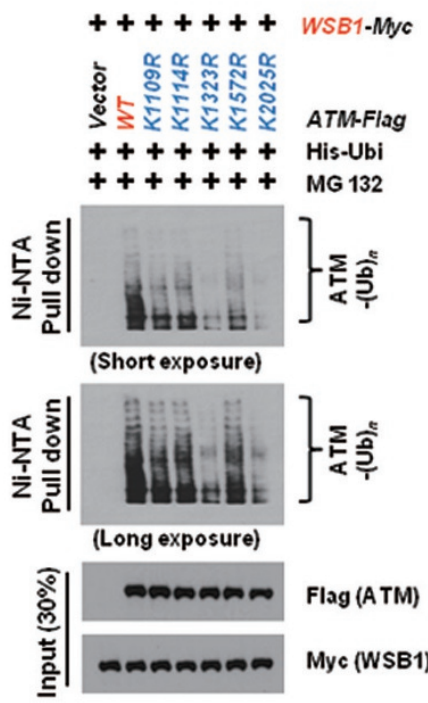

H

J
B

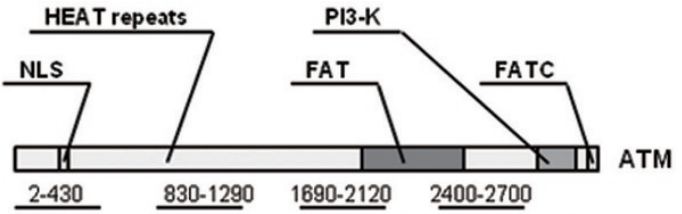

(1) $\frac{(2)}{400-860} \stackrel{\text { (3) }}{\frac{(4)}{1260-1720}} \stackrel{\text { (5) }}{\frac{(6)}{2090-2500}}{ }^{(7)} \frac{(8)}{2680-3056}$

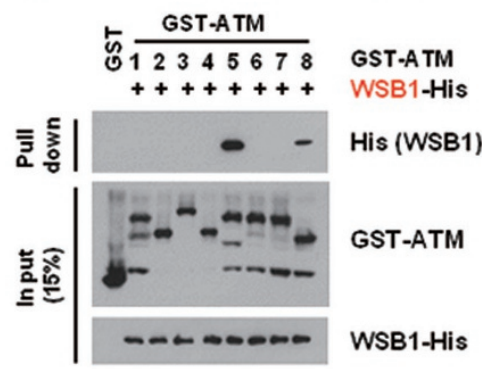

$\beta$-actin
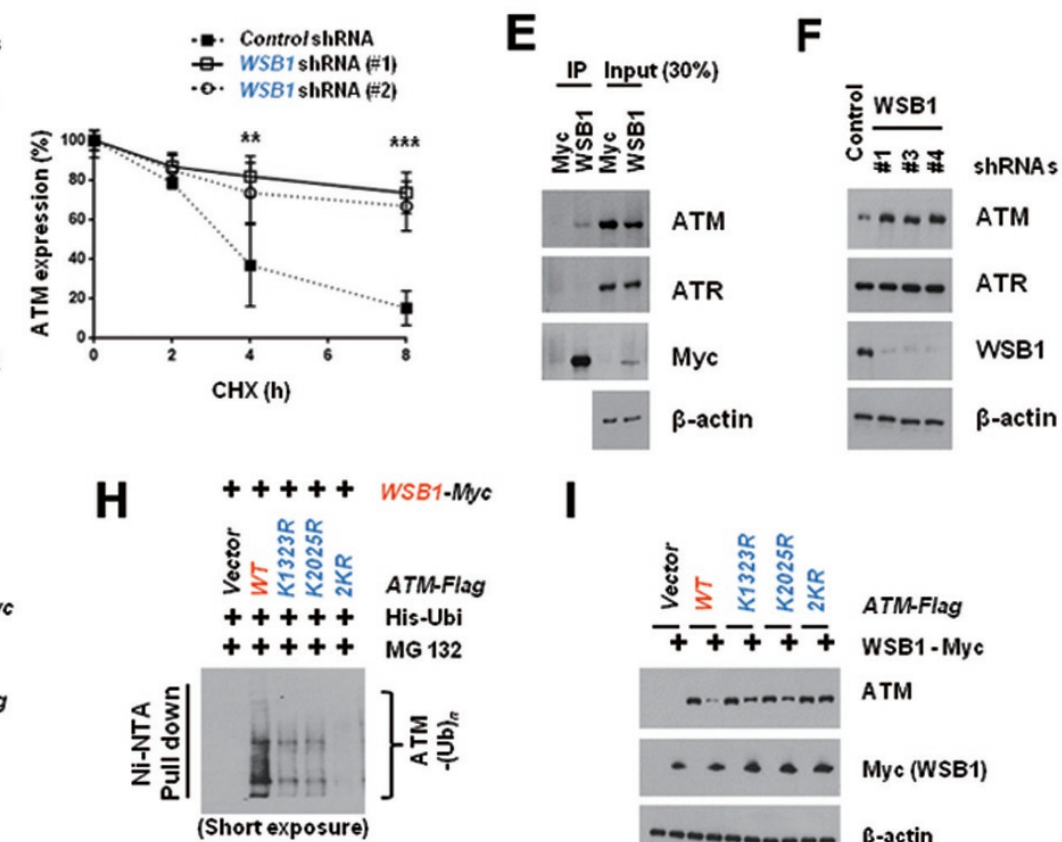

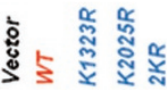

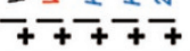

ATM-Flag

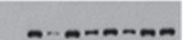

WSB1-Myc

$\ldots-\infty$ Myc (WSB1)

(Short exposure)
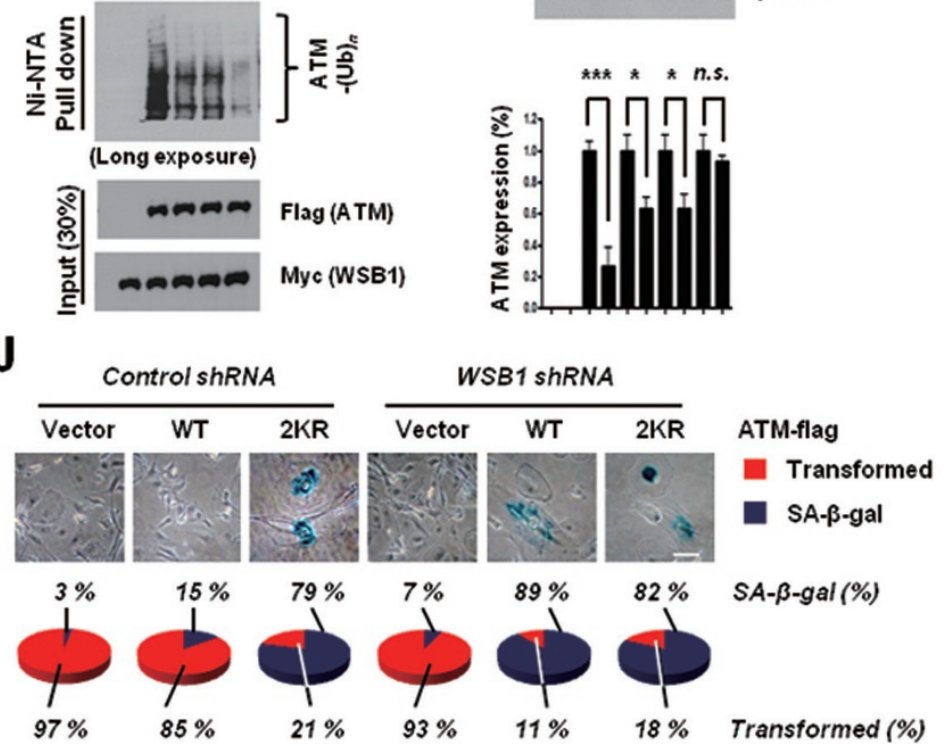
level of WT ATM was dramatically decreased by WSB1 overexpression. The expression of single KR mutants of ATM could also be decreased by WSB1 expression, although to a lesser extent than WT ATM. Importantly, the expression of 2KR was not affected by WSB1 expression (Figure 3I). We next tested whether reintroducing 2KR, but not WT ATM can confer resistance to WSB1-dependent escape of OIS. Both control cells and MEFs expressing WT ATM could be transformed by H-Ras ${ }^{\mathrm{V} 12}$ (Figure 3J). However, cells expressing 2KR were senescent and resistant to transformation by H-Ras. Furthermore, WSB 1 knockdown inhibited cell transformation induced by $\mathrm{H}-\mathrm{Ras}^{\mathrm{V} 12}$ in cells expressing WT ATM, and had no additional effect in cells expressing the $2 \mathrm{KR}$ mutant. All of the above results indicated that K1323 and K2025 are major ATM ubiquitination sites regulated by WSB1, and ubiquitination of these residues leads to ATM degradation.

\section{The SOCS domain of WSB1 is required for ATM interac-} tion and ubiquitination

We next determined how WSB1 interacts with ATM. WSB 1 contains several WD40 repeats and a SOCS domain [42]. WD40 repeats participate in various cellular functions, and the SOCS box is involved in protein degradation by the Elongin-Cullin-SOCS-box protein ubiquitin ligase complex [44-46]. To determine the region of WSB1 that is required for its interaction with ATM, we transfected Myc-tagged WSB1-deletion mutants into HEK 293 T cells and performed co-immunoprecipitation. We found that the C-terminal WD40 repeats (WD 6-7) and the SOCS domain of WSB1 were both required and sufficient for ATM interaction (Figure 4A and 4B). We further found that downregulation of ATM by WSB1 was dependent on the SOCS domain (Figure 4A and $4 \mathrm{C}$ ). Consistent with these results, we found that ubiquitination of ATM by WSB1 required the SOCS domain (Figure 4D). In addition, ATM was more stable in cells expressing the WSB1 mutant lacking the SOCS domain (Figure 4E). Together, these results indicate that WSB1 interacts with ATM via its WD40 repeats and SOCS domain and regulates ATM ubiquitination and degradation through the SOCS domain.

We reasoned that WSB1-induced ubiquitination and degradation of ATM could facilitate cellular transformation by oncogene through the suppression of DDR. Given the important function of the WSB1 SOCS domain in mediating ATM degradation, we investigated how the WSB1 mutant lacking the SOCS domain affects OIS. We infected MEFs with viruses encoding both $H$-Ras ${ }^{V 12}$ and WSB1 (WT or $\triangle$ SOCS) and examined OIS and cellular transformation. Consistent with Figure 1, co-expression of oncogenic Ras and WSB 1 promoted the escape from OIS and cell transformation (Figure $4 \mathrm{~F}$ and $4 \mathrm{G})$. Deletion of the SOCS domain abolished the ability of WSB1 to promote the escape from OIS, cell transformation, and abnormal cell proliferation (Figure 4F and 4G). ATM levels were decreased in cells expressing WT WSB1 but not in cells expressing mutant WSB1 ( $\triangle$ SOCS; Figure $4 \mathrm{H})$. In 3 -D culture systems, we obtained similar results. WT WSB1, but not $\triangle$ SOCS, together with $\mathrm{H}-\mathrm{Ras}^{\mathrm{V} 12}$ expression, promoted sphere formation (Figure 4I and 4J). Since senescenceassociated secretion phenotype (SASP) is a major characteristic of OIS $[3,47]$, we next examined the impact of WSB1 on SASP. We found that overexpression of WT WSB1 suppressed the expression of key SASP factors such as IL- 6 , IL-8, and IL- $1 \beta$ during OIS after $\mathrm{H}-\mathrm{Ras}^{\mathrm{V} 12}$ induction in IMR-90 ER:Ras cells (Figure 4K). However, WSB1 mutant lacking the SOCS domain did not affect SASP gene expression. These results together with Figure 2 suggest that one major mechanism by

Figure 3 WSB1 regulates ATM ubiquitination and degradation. (A) Co-immunoprecipitation (Co-IP) of endogenous ATM and WSB1 from extracts of HEK 293T cells. (B) Purified WSB1 were incubated with GST or equal amounts of GST-ATM fragments coupled to GSH sepharose. Proteins retained on sepharose were then blotted with the indicated antibodies. (C) Cells were transfected with the indicated constructs and then treated with MG132. Ubiquitinated proteins were pulled-down under denaturing conditions by Ni-NTA agarose and analyzed by immunoblot. (D) HEK 293T cells stably expressing control shRNA or WSB1 shRNA (\#1 and \#2) were treated with $\mathrm{CHX}(0.1 \mathrm{mg} / \mathrm{ml})$ and harvested at the indicated times. Cell lysates were then blotted with the indicated antibodies (left). Data (left) were quantified and normalized relative to the $\beta$-actin level using ImageJ program (right). ${ }^{* *} P<0.01$ and ${ }^{* * *} P<0.001$ versus control cells by one-way ANOVA. (E) Co-IP of WSB1 and ATM or ATR from extracts of HEK 293T cells. (F) Cells were transfected with WSB1 shRNAs and ATM or ATR levels were examined by immunoblot. $(\mathbf{G}, \mathbf{H})$ Cells were transfected with the indicated constructs and then treated with MG132. Ubiquitinated proteins were pulled-down under denaturing conditions by Ni-NTA agarose and analyzed by immunoblot. (I) ATM KO MEFs were transfected with the indicated constructs and ATM levels were then examined by immunoblot (top). Quantification of ATM protein expression levels are shown in top panel (bottom). ${ }^{*} P<0.05$ and ${ }^{* * *} P<0.001$ versus control cells by one-way ANOVA. (J) Cells were infected (or transfected by electroporation) with the indicated viruses (or constructs) and analyzed for senescence (top, SA- $\beta$-gal staining; bottom, quantification of senescent or transformed cells). 
A

B

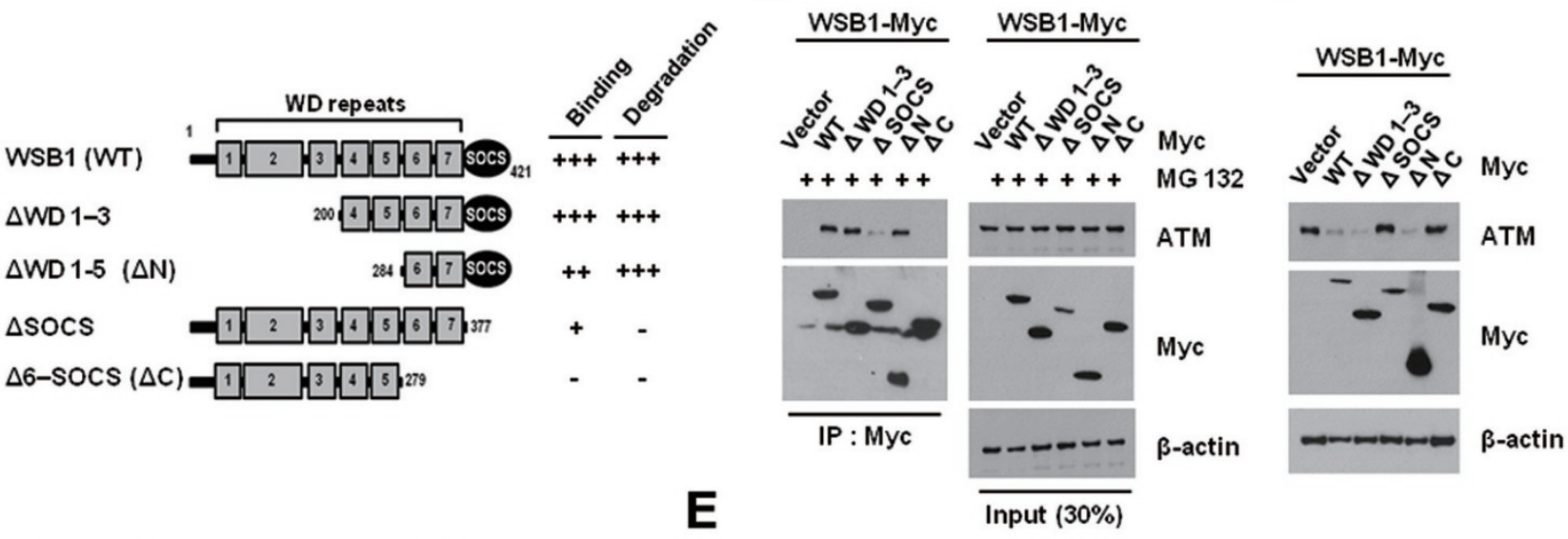

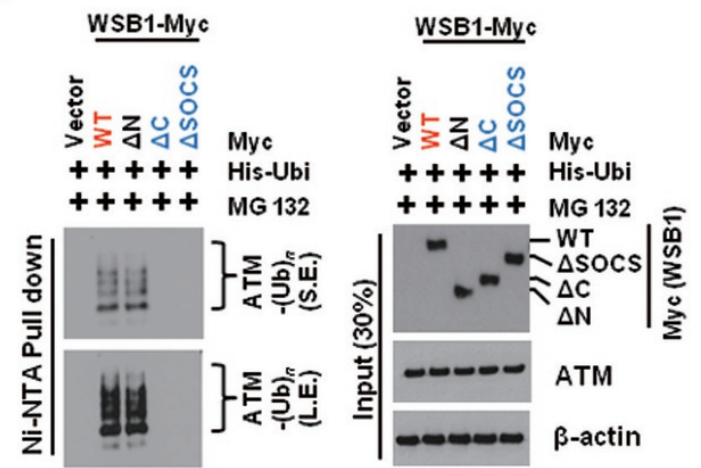

$\mathbf{F}$

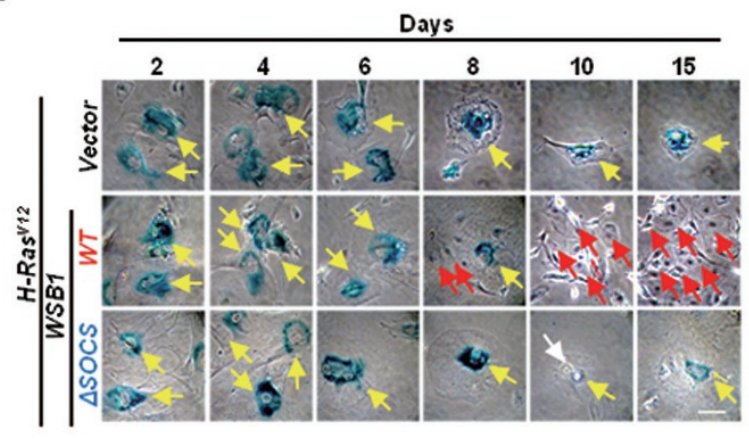

H

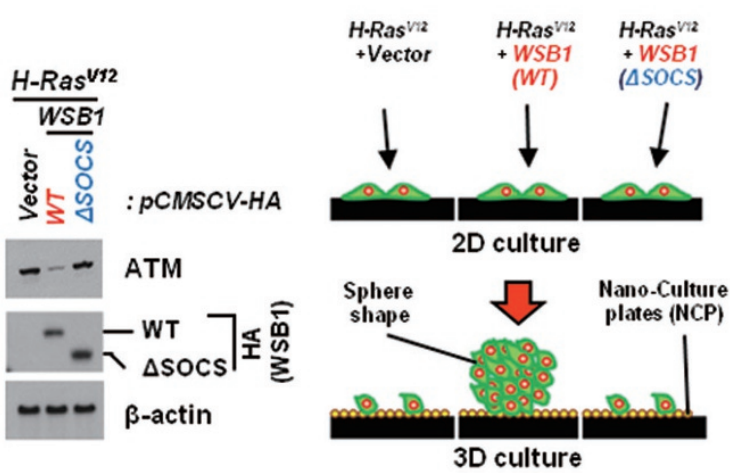

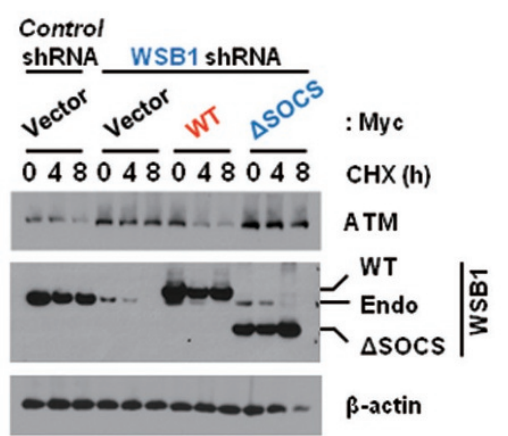

ShRNA $\quad$ WSB1-Myc

- $=\overline{\text { Control }}+$ Vector

- WSB1 + Vector

-WSB1 + WT

- WSB1 + $\triangle$ SOCS

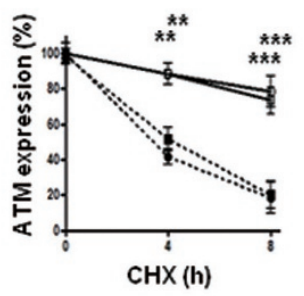

G

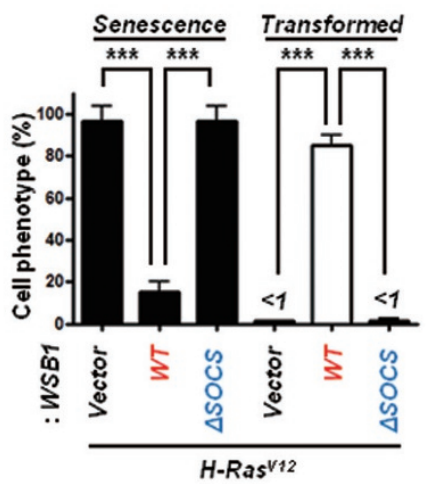

J

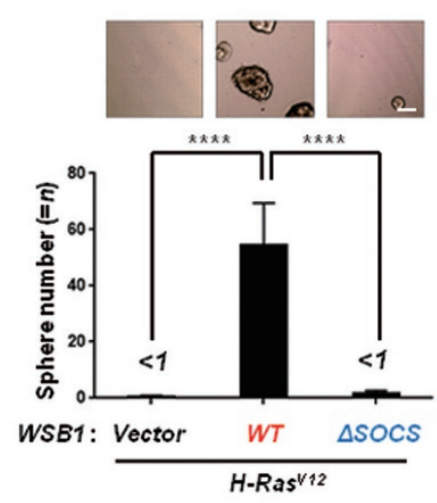

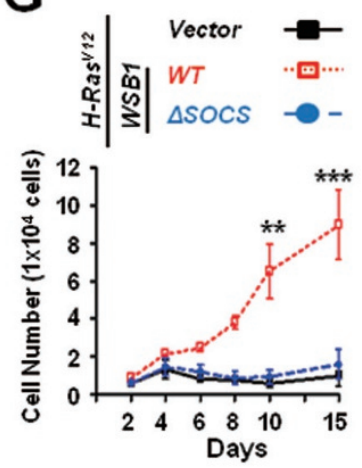

K

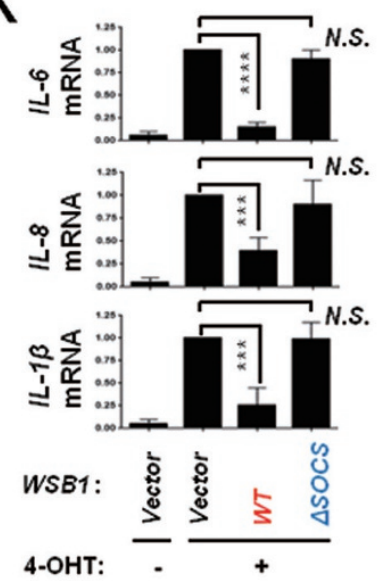


which WSB1 promotes the escape from OIS thus leading to cellular transformation is through inducing ATM ubiquitination and its subsequent degradation.

WSB1 phosphorylation by CDKs is required for its monomerization and activity

We next sought to determine the molecular mechanisms that regulate WSB1. For several E3 ligases, such as MDM2 and TRAF6, dimerization or oligomerization is involved in the regulation of activity [48-50]. Interestingly, we also found that WSB1 could form intermolecular interactions, and that the SOCS domain of WSB1 was required for its intermolecular interactions (Figure 5A). Using non-denaturing gel, we found that WSB1 existed as both homodimers and monomers (Supplementary information, Figure S3A). After H-Ras ${ }^{\mathrm{V} 12}$ induction in IMR-90 ER:Ras cells, to induce oncogenic stress, WSB1 homodimer changed into to the monomeric form in cells expressing WT WSB1 (Supplementary information, Figure S3A, second lane). We also found that WSB1 was in the active monomer form in MEFs expressing both $c-M y c$ and $H-$ Ras $^{V 12}$ (Supplementary information, Figure S3B). To study the mechanism of WSB1 monomerization, we examined WSB1 post-translational modifications. We found AKT, GSK3 $\beta$, CDK2, Plk 1, and Aurora B to be potential kinases of WSB1 using GPS2.12, a tool for predicting kinase-specific phosphorylation sites [51]. Therefore, we examined whether WSB1 monomerization is inhibited by kinase inhibitors. Only the CDK inhibitor (CDKI) Roscovitine was able to inhibit WSB1 monomerization (Supplementary information, Figure S3A). Since Roscovitine is a broad-range CDK inhibitor, we next used specific CDKIs to identify each CDK's impact on WSB1 modification and function. We used RO-3306 as CDK1 inhibitor, SNS-032 (BMS-387032) as CDK2 inhibitor and PD0332991 as CDK4 inhibitor. We found that WSB1 dimer/monomer transition was inhibited by CDK2 or CDK4 inhibitor (Figure 5B). The decrease in ATM protein levels was also inhibited by the CDK2 or CDK4 inhibitor (Figure 5C). However, CDK1 inhibitor had no effect. Interestingly, most oncogenes lead to abnormal cell cycle regulation and do so by directly or indirectly boosting the activities of cyclindependent kinases (CDKs) that function in the cell cycle. Furthermore, early studies suggest a role of CDK2 in suppressing oncogene- and/or stress-induced senescence $[52,53]$.

Therefore, we examined whether CDK regulates WSB 1 activity toward ATM. To test this, we first examined the ability of WSB1 to interact with CDKs. We found that endogenous WSB1 interacted with CDK2 and slightly with CDK4, but not with CDK1 (Figure 5D). We hypothesized that CDK-mediated phosphorylation of WSB1 might affect its dimerization. We next examined whether WSB1 is phosphorylated by CDK2. As shown in Figure 5E, CDK2 could directly phosphorylate WSB1 in vitro. As some WSB1 phosphorylation sites were revealed by mass spectrometry analysis (http://www. phosphosite.org) and GPS2.12, we analyzed whether these sites were phosphorylated by CDK. Of them, mutation of T380 to alanine (A) substantially reduced WSB1 phosphorylation (Figure 5F), suggesting that T380 may be a major site of phosphorylation mediated by CDK. In addition, Thr380 is within the SOCS domain and is highly conserved among vertebrates (Figure

Figure 4 WSB1 interacts with ATM via its WD40 repeats and SOCS domain and regulates ATM ubiquitination through the SOCS domain. (A) Diagrams of WT WSB1 and corresponding deletion mutants ( $\triangle \mathrm{WD} 1-3, \Delta \mathrm{WD} 1-5, \Delta S O C S$, and $\Delta 6$ SOCS) used in Co-IP experiments with ATM. Plus symbols indicate binding ability between different WSB1 mutants with ATM (left) or the degradation strength of the WSB1 mutants on ATM stability (right). (B) Cells were transfected with indicated plasmids and then treated with MG132. Cells were collected for immunoprecipitation (IP)-immunoblot analysis. (C) Cells were transfected with indicated plasmids and the ATM levels were examined by immunoblot. (D) Cells were transfected with the indicated constructs and then treated with MG132. Ubiquitinated proteins were pulled-down under denaturing conditions by Ni-NTA agarose and analyzed by immunoblot. S.E. indicates short exposure; L.E., long exposure. (E) Cells were treated with $\mathrm{CHX}(0.1 \mathrm{mg} / \mathrm{ml})$ and harvested for different hours. ATM levels were then examined by immunoblot (left). Right panel shows quantification of ATM levels. The results represent the means ( \pm SE) of three independent experiments performed in triplicate. ${ }^{* *} P<0.01$ and ${ }^{* * *} P<0.001$ versus control cells by one-way ANOVA. Data (left) were quantified and normalized relative to the $\beta$-actin level using ImageJ program (right). (F, G) Primary MEFs were infected with the indicated constructs and were analyzed for senescence by SA- $\beta$-gal staining assay (F) and cell-proliferation assay (G). Scale bar, $10 \mu \mathrm{m}$. Yellow arrows indicate SA- $\beta$-gal stained cells; red arrows, transformed cells; white arrow, death cells. The results represent the means $\left( \pm \mathrm{SE}\right.$ ) of three independent experiments performed in triplicate. ${ }^{* *} P<0.01$ and ${ }^{* * *} P<0.001$ versus control cells by one-way ANOVA. (H) Cells as in F were collected for immunoblot analysis. (I, J) Primary MEF cells as in $\mathbf{F}$ were applied on 3-D organoid cultures system. Schematic of the experiments (I) and sphere number (J) in 3-D organoid culture. ${ }^{* * * *} P<0.0001$ versus control cells by one-way ANOVA. (K) IMR-90 ER:Ras-inducible cells were infected with indicated viruses, and H-Ras was inducted by 4-OHT. Cells were analyzed for 'cytokine' mRNAs by real-time PCR analysis. 
A

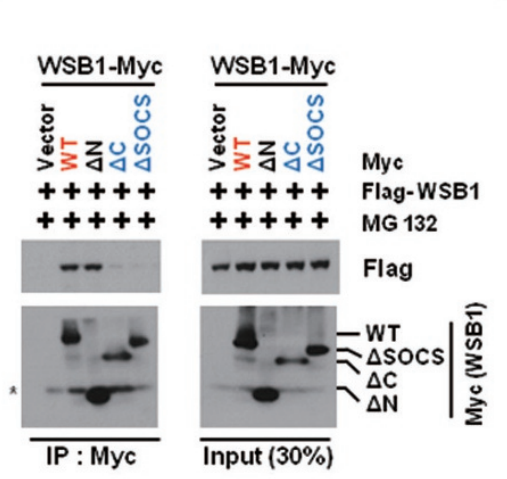

D

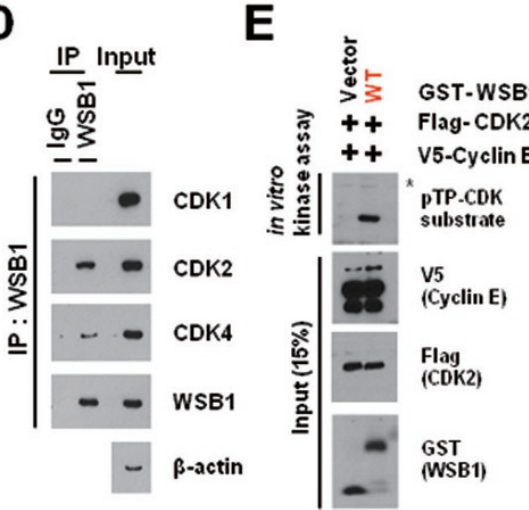

J

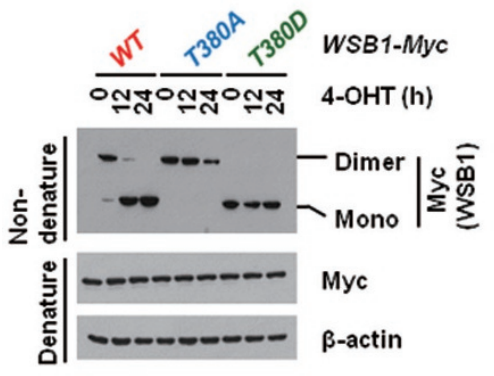

M

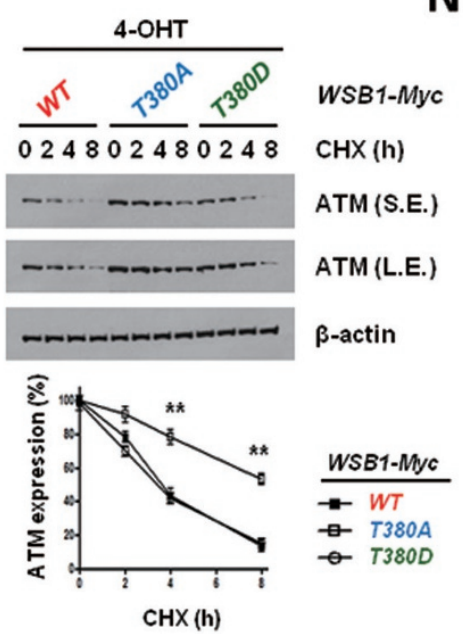

$\mathbf{N}$
B

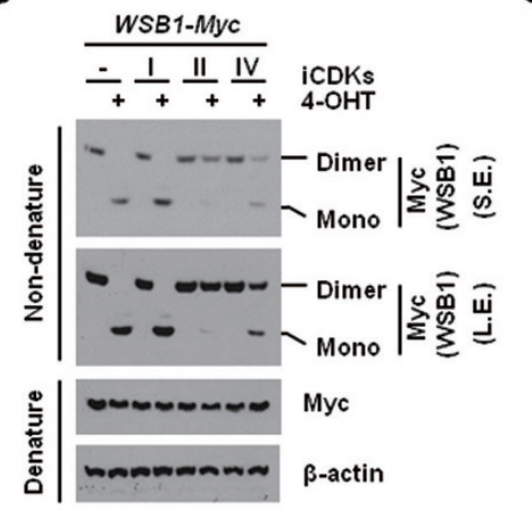

$\mathbf{F}$
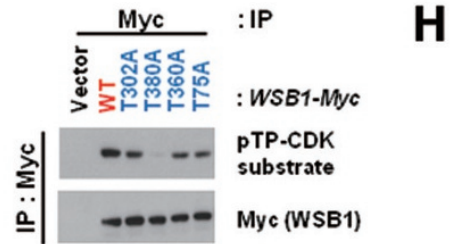

흘 니 - - - $\beta$-actin

$\mathbf{G}$

$373 \quad 387$

Hum an GSVYFWATPRQVPSL

Mouse GSVYFWATPRQVPSL

Rat GSVYFWATPRQVSSL

Monkey GSVYFWATPRQVPSL

Dog GSVYFWATPRQVPSL

K

1

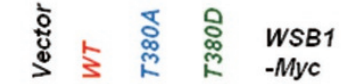
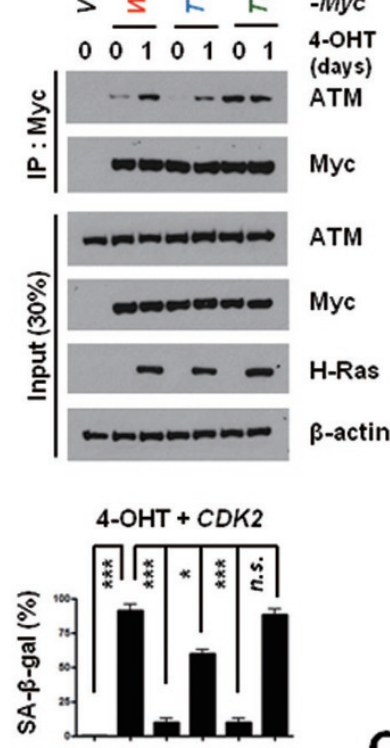

C
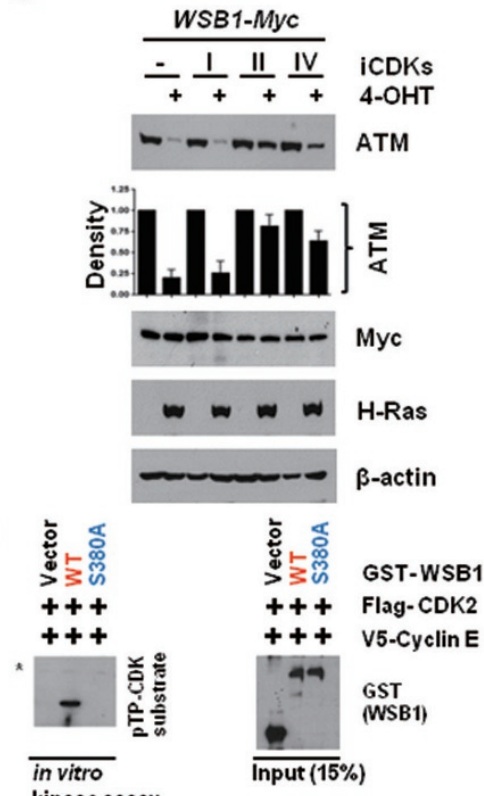

IgG WSB1 :IP

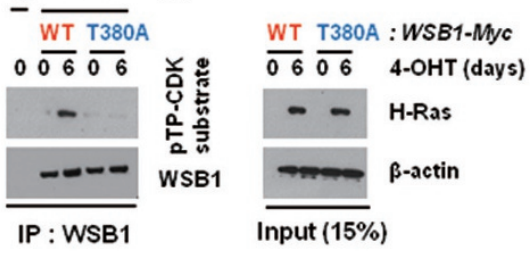

$\mathbf{L}$
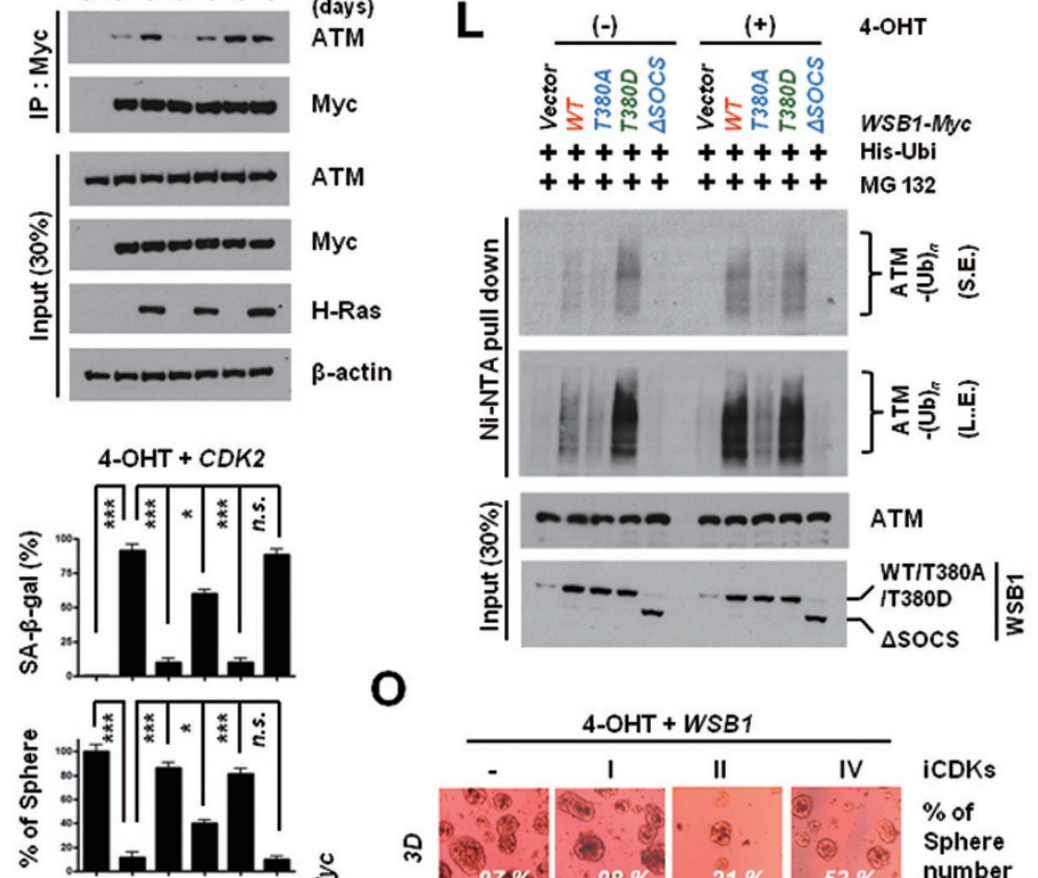

O
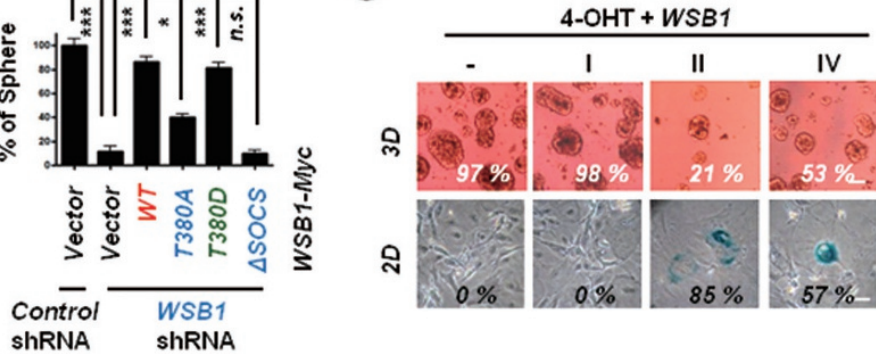

iCDKs $\%$ of

Sphere

number

SA- $\beta-g a l$

(\%) 
5G). As shown in Figure 5H, mutating Thr380 to Ala abolished CDK2-mediated phosphorylation of WSB1 in vitro. Furthermore, we found that mutation of T380 (T/A) abolished WSB1 phosphorylation induced by Ras expression (Figure 5I), suggesting that T380 is the major CDK phosphorylation site of WSB1 induced by Ras. Previous studies have shown that the Ras-MAPK pathway could upregulate CDK activities through several mechanisms including the inhibition of CDKIs and phosphorylation of CDKs at activating residues [54-58]. Many other oncogenic signals could also activate CDKs.

We next examined how T380 phosphorylation would affect WSB1 function. Because CDKs are involved in the regulation of WSB1 dimerization (Figure 5B and Supplementary information, Figure S3A), we tested whether the phosphorylation of T380 can regulate WSB1 dimerization (Figure 5J). We found that in cells expressing the T380A mutant, WSB1 maintained the dimeric form before and after oncogenic stress. On the other hand, T380D only existed in the monomeric form with or without oncogenic stress (Figure 5J). These results suggest that oncogenic stress induces a transition of WSB1 dimer to monomer in a T380 phosphorylationdependent manner. These results also imply that the T380A mutation did not completely disrupt WSB1 structure, since WSB1 T380A was still able to form a dimer similar to WT WSB1 under unstressed conditions.

We next tested whether T380 phosphorylation has any effect on ATM interaction and ubiquitination. We found that oncogenic stress increased the interaction between WSB1 and ATM (Figure 5K). The T380A mutant had decreased binding to ATM with or without oncogenic stress, while the T380D mutant had constitutively strong binding to ATM. These results suggest that the dimer form of WSB1 has decreased ATM binding, probably due to the masked SOCS domain. Consistent with this, we found that in unstressed conditions, ATM was highly ubiquitinated in cells expressing the T380D mutant, while WT WSB1 and the T380A mutant had low E3 ligase activity. Upon oncogenic stress, ATM ubiquitination increased in cells expressing WT WSB1 to a level comparable to that in cells expressing T380D.

Figure 5 WSB1 phosphorylation by CDKs activates WSB1. (A) Myc-tagged WT WSB1 and the corresponding mutants $(\Delta \mathrm{WD} 1-5[\Delta \mathrm{N}], \Delta 6$-SOCS $[\Delta \mathrm{C}]$, and $\Delta \mathrm{SOCS})$ were used in Co-IP experiments with Flag-tagged WT WSB1. *indicates a nonspecific band. (B, C) Cells were collected for immunoblot analysis. IMR-90 ER:Ras-inducible cells were infected with viruses encoding WT WSB1, and H-Ras was induced by 4-OHT. Cells were pre-treated with indicated inhibitors. WSB1 dimerization was then examined under non-reducing (top, B) or reducing condition (bottom, B). Data (top, C) were quantified and normalized relative to the $\beta$-actin level using ImageJ program (middle, C). CDKs inhibitors. RO-3306 (9 $\mu$ M), CDK1 inhibitor; SNS-032 (BMS-387032, $0.1 \mu \mathrm{M})$, CDK2 inhibitor; PD0332991 (0.5 $\mu \mathrm{M})$, CDK4 inhibitor. (D) Co-IP of endogenous CDKs and WSB1 from extracts of HeLa cells. (E) HEK 293T cells were transfected with Flag-CDK2 and V5-Cyclin E. The cell lysates were immunoprecipitated with anti-Flag antibody. The protein conjugated to the beads were eluted with Flag peptides and subjected to in vitro kinase assay. GST and GST-WSB1 expressed in bacteria were purified and subjected to kinase assay as substrates. The phosphorylation of WSB1 by CDK2 was examined with indicated antibodies. *indicates a nonspecific band. (F) IMR-90 ER:Ras-inducible cells were infected with viruses encoding WT WSB1, T302A, T380A, T360A, or T75A, and H-Ras was induced by 4-OHT. WSB1 phosphorylation was then examined with indicated antibodies. (G) Comparison of the sequences surrounding T380 of WSB1 orthologs. Thr380 is shown in red. (H) GST and GST-WSB1 WT or mutant (T380A) expressed in bacteria were purified and subjected to in vitro kinase assay with CDK2+Cyclin E (Millipore). The phosphorylation of WSB1 by CDK2 was examined using anti-phospho-CDKs substrate antibody. *indicates a nonspecific band. (I) IMR-90 ER:Ras-inducible cells were infected with viruses encoding WT WSB1 or T380A, and H-Ras was inducted by 4-OHT for indicated times. WSB1 phosphorylation was then examined with indicated antibodies. (J) IMR-90 ER:Rasinducible cells were infected with viruses encoding WT WSB1, T380A, or T380D, and H-Ras was induced by 4-OHT for indicated times. WSB1 dimerization was then examined under non-reducing (top) or reducing condition (bottom). (K) Cells as in $\mathbf{J}$ were treated with MG132, and the interaction between WSB1 (WT, T380A, or T380D) and ATM was examined IPimmunoblot analysis. (L) Cells were transfected with the indicated constructs and then treated with MG132. Ubiquitinated proteins were pulled-down under denaturing conditions by Ni-NTA agarose and analyzed by immunoblot. (M) Cells as in $\mathbf{L}$ were treated with $\mathrm{CHX}(0.1 \mathrm{mg} / \mathrm{ml})$ and harvested at the indicated times. ATM levels were then examined. Data (top) were quantified and normalized relative to the $\beta$-actin level using ImageJ program (bottom). The results represent the means $( \pm$ $\mathrm{SE}$ ) of three independent experiments performed in triplicate. ${ }^{* *} P<0.01$ versus control cells by one-way ANOVA. (N) CDK2 expressing IMR-90 ER:Ras-inducible cells were applied senescence by SA- $\beta$-gal staining assay in a 2-D culture system (top) and cellular sphere formation in a 3-D organoid culture system (bottom). Quantification of senescence by SA- $\beta$-gal staining assay (top) and sphere number in 3-D organoid culture (bottom), stained as in Supplementary information, Figure S3C. ${ }^{*} P<$ 0.05 and ${ }^{* * *} P<0.001$ versus control cells by one-way ANOVA. (O) ER:Ras-inducible cells were infected with the indicated viruses and were pre-treated with indicated inhibitors. The cells were assayed by the 3-D organoid cultures system (top) and SA- $\beta$-gal staining (bottom). Quantification of sphere number (\%) in 3-D culture (top) and senescence (\%) by SA- $\beta$-gal staining assay (bottom). Scale bar, $10 \mu \mathrm{m}$. 
Less induction of ATM ubiquitination was found in cells expressing the T380A mutant (Figure 5L). Next, we tested whether phosphorylation of this site has any effect on ATM stability. We expressed WSB1 WT or WSB 1 mutant forms (T380A or T380D) in IMR-90 ER:Ras cells and measured ATM stability following Ras induction. Interestingly, we found that ATM was more stable in cells expressing the T380A mutant (Figure 5M). T380D, which mimics T380 phosphorylation, showed similar effects as WT WSB1. These results suggest that T380 phosphorylation might be important for WSB1 E3 ligase activity.

Given the important function of CDKs in activating WSB1 to mediate ATM degradation, we investigated how CDKs affect OIS through WSB1. We focused on CDK2, as it showed higher binding to endogenous WSB1. We infected CDK2-expressing IMR-90 ER:Ras cells with viruses encoding WSB1 shRNA, and examined OIS in a 2-D culture system and cellular sphere formation in a 3-D culture system. We found that co-expression of oncogenic Ras and CDK2 promoted the escape from OIS (Figure 5N). Depletion of WSB1 considerably increased OIS and decreased the cell-sphere number induced by oncogenic Ras plus CDK2 (Figure 5N and Supplementary information, Figure S3C). Ectopic expression of WT WSB1 and WSB1 (T380D), but not WSB1 $(\triangle$ SOCS), restored the ability of oncogenic Ras together with CDK2 to induce sphere formation or inhibit OIS (Figure $5 \mathrm{~N}$ and Supplementary information, Figure S3C). The expression of WSB1 (T380A) had partial effects on the inhibition of OIS or induction of sphere formation. ATM expression was decreased in CDK2-expressing Ras-induced IMR90. However, depletion of WSB1 substantially increased ATM level (Supplementary information, Figure S3D). We found similar results with CDK4 expression, but not CDK1 (Supplementary information, Figure S3E). Furthermore, CDK2 or CDK4 inhibitor suppressed WSB1-induced sphere formation or escape from OIS (Figure 5O).

Previous studies suggest that the CDK inhibitor p21 is upregulated during cellular senescence and overexpression of $\mathrm{p} 21$ leads to a senescence-like growth arrest [59-62]. Given the function of $\mathrm{p} 21$ as a CDK2 inhibitor, it is likely that p21 may have an effect on the phosphorylation of WSB1. To test this, we overexpressed p21 and found that WSB1 phosphorylation and WSB1 dimer/monomer transition were inhibited by $\mathrm{p} 21$ overexpression (Supplementary information, Figure S3F-S3H). Furthermore, WSB1's effects on OIS suppression and sphere formation were also inhibited by $\mathrm{p} 21$.

These results suggest that WSB1 is phosphorylated by CDKs at T380, and T380 phosphorylation induces a transition of WSB1 dimer to monomer. Monomeric WSB1 exhibits stronger binding and better E3-ligase activity toward ATM. Therefore, oncogenic stress could activate WSB1 at both a transcriptional level (e.g., Myc) and a post-translational level (e.g., CDK).

\section{WSB1 promotes tumorigenesis}

Since ATM is an upstream activator of the DDR, which acts as an important barrier of tumorigenesis, and WSB1 inhibits ATM function by ubiquitinating ATM, we hypothesized that WSB1 functions as an oncogene to suppress OIS and promote tumorigenesis. To further test this hypothesis and to investigate the functional relevance of the WSB1-ATM axis in malignancy in vivo, we performed tumorigenesis assay in mouse models. MEFs with overexpression of both H-Ras ${ }^{\mathrm{V} 12}$ and WSB1 exhibited high tumorigenic potential, as demonstrated by subcutaneous xenografting into athymic nude mice (Supplementary information, Figure S4A and S4B). Next, we examined the effect of WSB1 and the SOCS domain on tumor formation using subcutaneous xenografts. We found that depletion of WSB1 considerably decreased tumor formation or cell sphere number induced by oncogenic Ras (Figure 6A and 6B and data not shown). Ectopic expression of WT WSB1, but not WSB1 $(\triangle \mathrm{SOCS})$, restored the ability of oncogenic Ras to induce tumorigenesis (Figure 6A and 6B). We next asked whether WSB1 remains in the active monomer forms during in vivo MEF transformation. We found that WSB1 was in the active monomer form in xenograft tumor tissues (Supplementary information, Figure S4C). Furthermore, to establish the effect of WSB1 as ATM's E3 ligase during tumorigenesis, we used $A T M^{\prime-}$ immortal MEFs reconstituted with WT ATM or ATM Ub mutants (K1323R, K2025R, K1323R/K2025R) (Figure 6C). We found that the single Lys mutation of ATM (K1323R or K2025R) partially decreased tumor formation induced by WSB1, and the 2KR mutation of ATM considerably decreased tumorigenic potential of WSB1. These results support that ubiquitination of ATM by WSB1 contributes to WSB1's tumorigenic potential.

To test whether CDK2/CDK4 inhibitor would suppress tumorigenesis by targeting the WSB1-WT ATM axis, we treated the xenografts with CDK2 or CDK4 inhibitor (Figure 6D). We found that CDK2 or CDK4 inhibitor decreased tumor formation induced by WSB1 in xenograft models expressing WT ATM and slightly decreased tumor formation for those expressing the single mutant version of ATM (K1323R or K2025R). However, these inhibitors had no effect on tumor formation for cells expressing the 2KR mutant ATM (Figure 6D). 
A

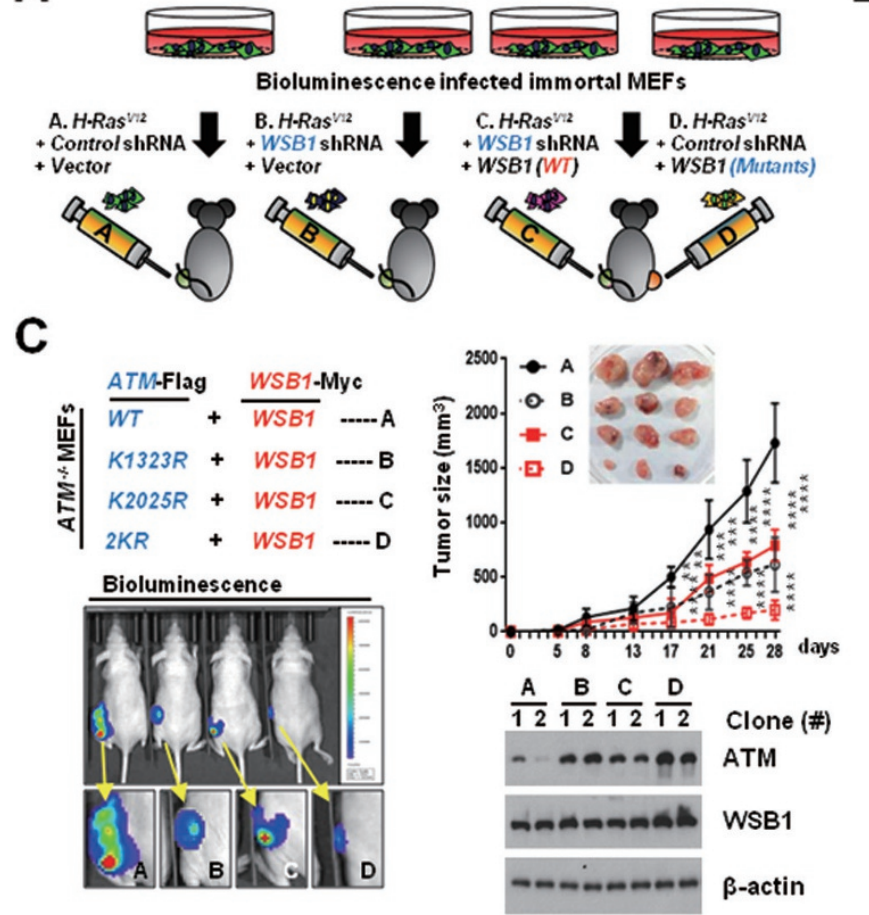

B

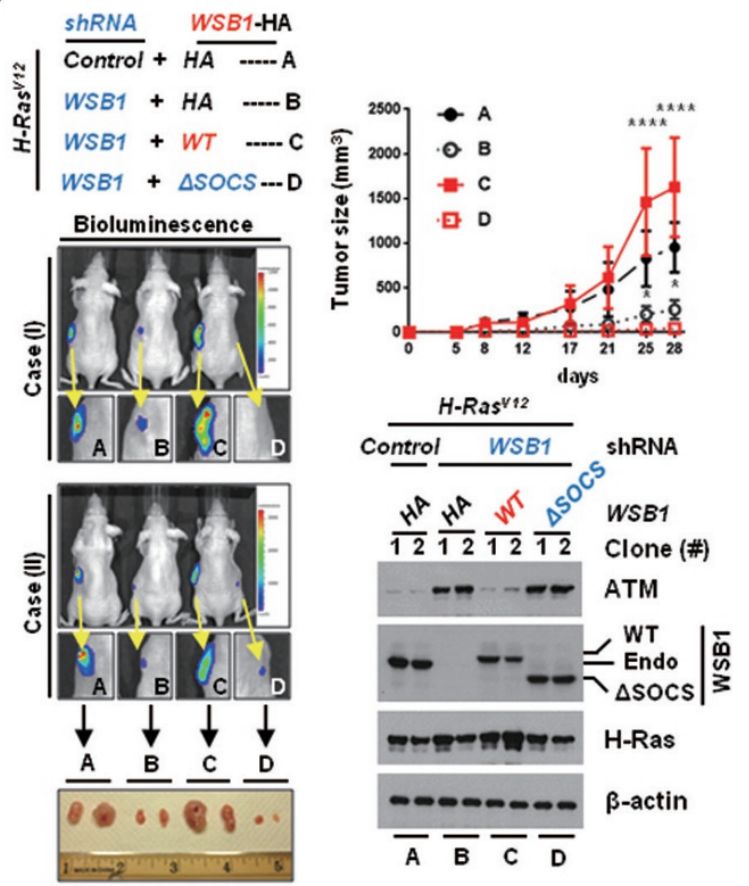

D

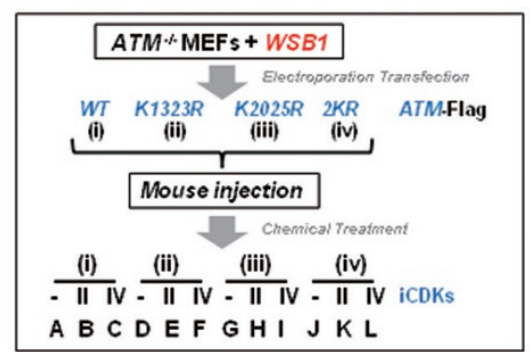

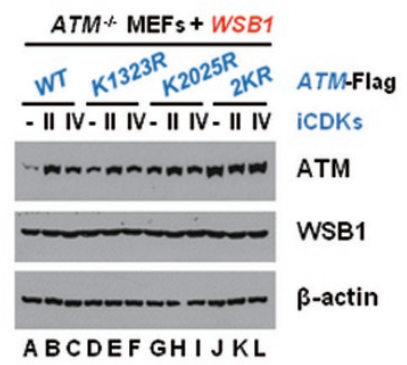

E

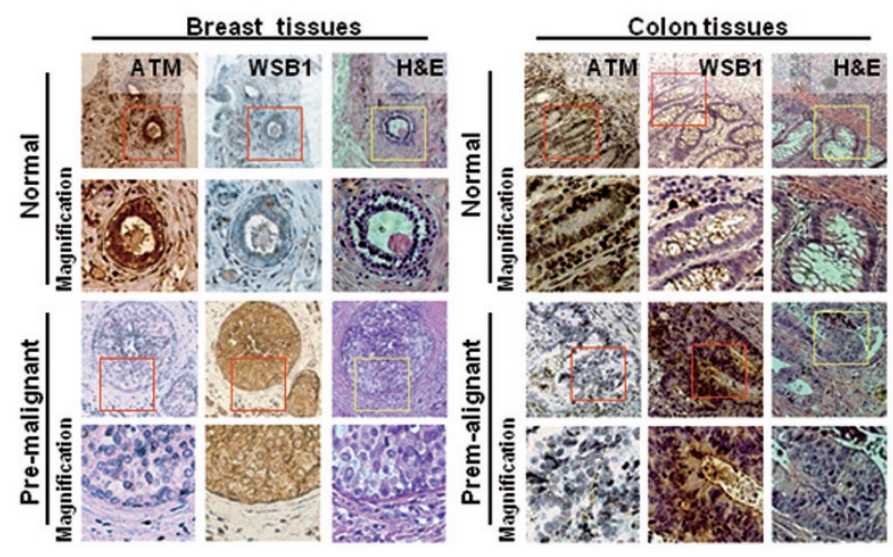

ATM*MEFs + WSB1
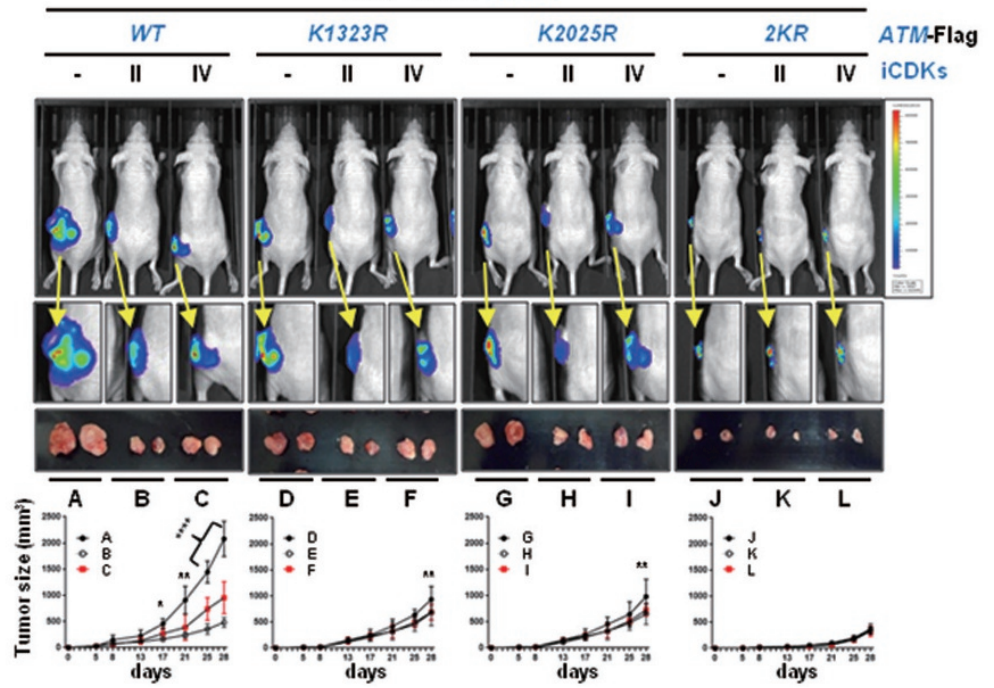

$\mathbf{F}$

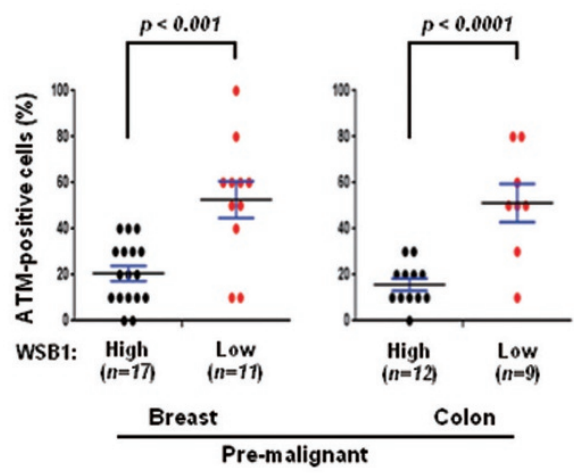


Finally, we examined human cancer cell lines and clinical samples for WSB1 expression. We found a negative co-relation between WSB1 and ATM level in GBM xenografts, pancreatic cancer cell lines, and nonsmall cell lung cancer (NSCLC) cell lines (Supplementary information, Figure S4D-S4F). To examine the expression of WSB1 and ATM in human tumor tissues, we performed immuno-histochemical staining of WSB1 and ATM using published antibodies for IHC [26, 34, 63, $64]$ in breast and colon pre-malignant samples (Figure $6 \mathrm{E})$. We found that WSB1 expression was higher in precancer lesions compared with normal adjacent tissues, while ATM expression generally negatively correlated with that of WSB1 (Figure 6E and 6F). These results are consistent with the negative regulation of ATM by WSB1 in human cancers.

\section{Discussion}

In the present study, we found an important mechanistic link between WSB1 and ATM, and our data support the model presented in Figure 7 in which WSB1 induces ATM degradation through its E3 ligase function. We also show that CDKs phosphorylate WSB1 and enhance WSB 1 activity against ATM. We propose that the regulation of ATM by WSB1 is a mechanism for cells to overcome OIS, which acts as a main tumorigenesis barrier in pre-malignant lesions. In primary cells expressing oncogenic Ras alone, the basal level of WSB 1 is low and might not be able to sufficiently remove ATM to overcome OIS. However, when WSB1 is overexpressed in primary cells, or in cells expressing two or more oncogenes (e.g., c-Myc), WSB1 levels will reach a threshold sufficient to downregulate ATM and help overcome OIS to promote cell transformation. Our findings provide a potential mechanism by which cells escape from OIS during tumorigenesis.

ATM plays a critical role in the DDR. An intact and effective DDR is essential for the maintenance of genomic stability and acts as a critical barrier to suppress tumor formation. Inactivation of ATM may result in predisposition to tumors and has been associated with lung, breast, and prostate cancer, as well as hematologic cancers such as T-cell prolymphocytic leukemia, B-cell chronic lymphocytic leukemia, and mantle cell lymphoma [65-67]. Furthermore, there are high rates of germline mutation or loss of the ATM gene in hematologic cancers [68]. Previous studies also suggest that the ATM protein level was reduced in several cancers, including breast cancer, glioblastoma, oral squamous cell carcinoma, gastric cancer, and colorectal cancer [26, 69-73]. However, the cellular mechanisms regulating ATM expression at the post-transcription level are poorly understood. Understanding how ATM is regulated at the post-transcription level will have important implications in understating tumorigenesis, especially tumor initiation in premalignant lesions. For example, in breast cancer, ATM methylation, hypermethylation of the ATM promoter, and ATM copy number loss have been shown to be associated with lower steady-state $A T M$ mRNA levels [41, 74, 75]. However, only a very small proportion of breast cancer is associated with low ATM mRNA levels, while downregulation of ATM is found at relatively high frequency (40\%-75\% cases) $[40,69$, $76,77]$. Notably, our results provide a new mechanism by which ATM is downregulated in human cancers through post-translational modification by WSB1. In support of the negative regulation of ATM by WSB1,

Figure 6 WSB1 regulation is a driving event in tumorigenesis. (A, B) Immortal MEFs were infected with the indicated constructs and used in xenograft models. The cells were applied on mice models. Schematic of the experiments (A) and tumor formation in xenograft (B). (B) Bioluminescence images of tumor formation in xenograft mice (left). Tumor volumes $\left(\mathrm{mm}^{3}\right)$ were measured at the indicated times after injection (right). Immunoblotting of WSB1 and ATM (bottom right). $n=10$ per group. ${ }^{*} P<0.05$ and ${ }^{* * * *} P<0.0001$ versus control cells by two-way ANOVA. (C) ATM KO MEFs were transfected with the indicated constructs and injected into nude mice. Left panel: bioluminescence images of tumor formation in xenograft were taken. Right top panel: tumor volumes $\left(\mathrm{mm}^{3}\right)$ were measured at the indicated times after injection. Right bottom panel: immunoblotting of WSB1 and ATM. $n=5$ per group. ${ }^{* *} P<0.01$, ${ }^{* * *} P<0.001$, and ${ }^{* * * *} P<0.0001$ versus control cells by twoway ANOVA. (D) ATM KO MEFs were transfected with the indicated constructs by electroporation and then injected into nude mice. And mice were treated with indicated inhibitors. Schematic of the experiments (top left) and tumor formation in xenograft (right). Bioluminescence images of tumor formation in xenograft were taken (top right). Tumor volumes $\left(\mathrm{mm}^{3}\right)$ were measured at the indicated times after injection (bottom right). CDKs inhibitors: SNS-032 (BMS-387032, intraperitoneal injection, 15 mg/ kg body weight every 3 days), CDK2 inhibitor; PD0332991 (oral administration, $150 \mathrm{mg} / \mathrm{kg}$ body weight daily), CDK4 inhibitor. Immunoblotting of WSB1 and ATM (bottom left). $n=5$ per group. ${ }^{*} P<0.05$, ${ }^{* *} P<0.01$, and ${ }^{* * *} P<0.0001$ versus control cells by two-way ANOVA. (E, F) Immunohistochemical staining of WSB1 and ATM protein in patient tissue samples (breast or colon). Representative microscopy images of WSB1 and ATM (E), and WSB1 and ATM expression correlation (F) in 28 human breast pre-cancer and 21 colon pre-cancer tissues with normal lesion. The $P$-value was calculated by the Student's t-test. 


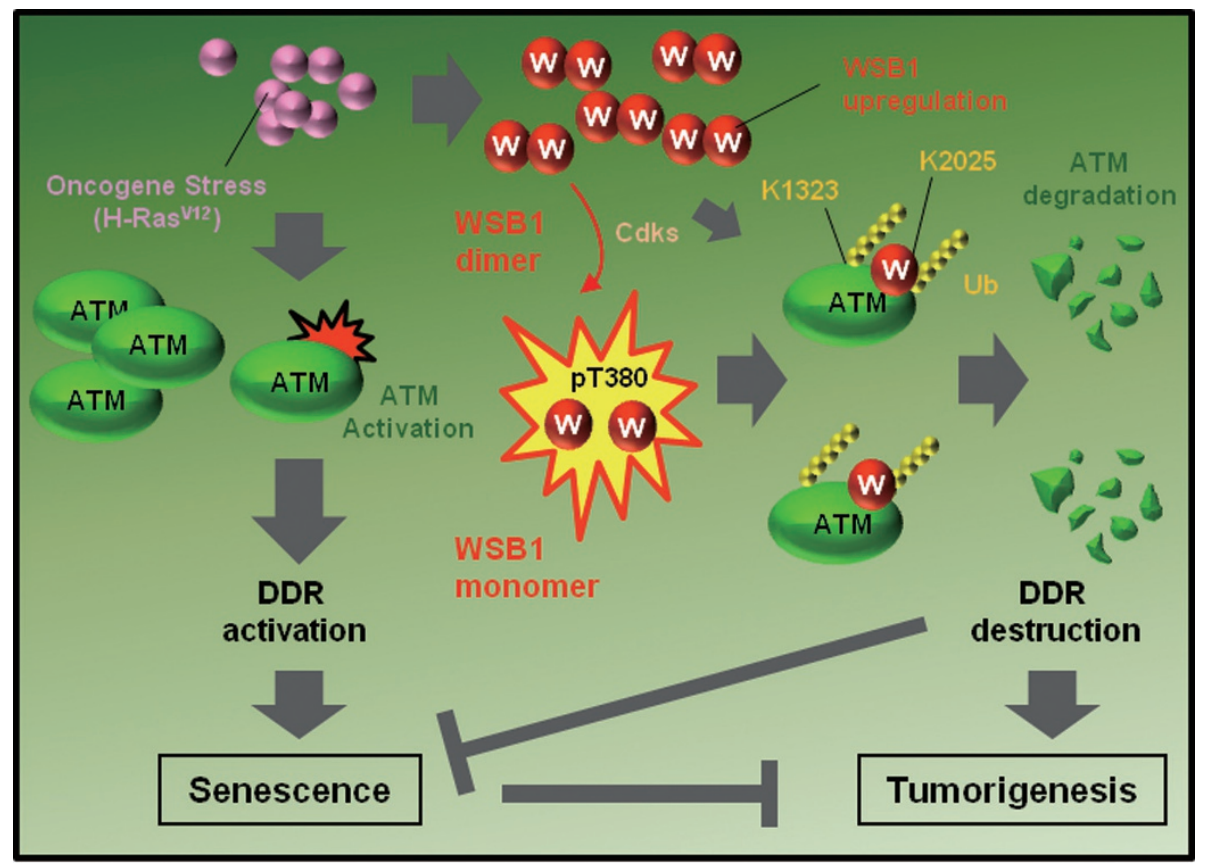

Figure 7 The working model. In typical conditions, oncogenic stress induces the DDR and oncogene-induced senescence, acting as the barrier of tumorigenesis. Oncogenic stress leads to WSB1 upregulation, which interacts with ATM and consequently degrades ATM. Meanwhile, CDKs are upstream kinase of WSB1. WSB1 is phosphorylated by CDKs, which creates WSB1 monomerization to enhance WSB1 activity toward ATM stability. WSB1 upregulation and activation could help ATM degradation, resulting in escape from OIS and breakage of the tumor barrier.

we found a negative co-relation between WSB1 and ATM level in not only GBM patient-derived xenografts, pancreatic cancer cell lines, and NSCLC cell lines, but also in breast and colon cancer patient samples (Figure $6 \mathrm{E}$ and $6 \mathrm{~F}$ and Supplementary information, Figure S4DS4F). Furthermore, WSB1 has been reported to be one of the proteins positively correlated with tumor incidence and tumor development in several cancers, including breast cancer $[32,78]$. Therefore, reduced steady-state ATM protein levels in WSB1-expressing cells might be responsible for compromised DDR, OIS escape, and genomic instability during tumor formation.

In summary, we have demonstrated that WSB1 is a tumor-promoting factor that mediates ATM degradation and helps overcome the main barrier of tumor formation. These findings suggest that pathologic upregulation of WSB1 in pre-malignant lesions may eventually contribute to the malignant phenotype of certain tumors. Indeed, WSB 1 overexpression is found in multiple cancer tissues and the WSB 1 gene is upregulated in premalignant lesions. Thus, our data support a model in which WSB1 helps the destruction of the DDR tumorigenesis barrier in pre-malignant lesions.

\section{Materials and Methods}

\section{Cells, cell lines, and reagents}

All cell lines were sourced from commercial venders. Human embryonic kidney (HEK) 293T, 293, and HeLa cervix carcinoma cells were cultured in Dulbecco's modified Eagle's media (DMEM, Gibco-Invitrogen, Grand Island, NY, USA). Twelve GBM xenografts from patients, normal human lung fibroblast (WI-38) cells, six human lung cancer (4 adenocarcinoma, H1437, H522, H1650, and A549; 2 large cell carcinoma, H460 and H1299) cell lines, and nine human pancreatic cancer (BxPC3, Hup-T3, MiaPaca, Panc1, Pan 04.03, ASPC1, CAPAN1, and CFPAC1) cells were maintained in Eagle's minimal essential media (EMEM) or RPMI 1640 (Gibco-Invitrogen). The human lung fibroblast IMR90 and WI-38 cells were obtained from the American Type Culture Collection (ATCC, Manassas, VA, USA), and cells ranging from 29 to 34 in population-doubling level were used. ER-RAS-IMR-90 cells were kindly provided by $\operatorname{Dr}$ Z Zhang (Mayo Clinic, USA). For ER-RAS induction, ER-RAS-IMR-90 cells were treated with $100 \mathrm{nM}$ of 4-hydroxytamoxifen (4-OHT) and maintained in 4-OHT-containing medium until harvesting.

MEFs were isolated from embryonic day 11.5-13.5 (E11.5-E13.5) by uterine dissection for individual embryos. Each embryo was rinsed softly with $1 \times \mathrm{PBS}(\mathrm{pH} 7.2)$, followed by removal of the mouse embryonic internal organ. The embryo body was transferred to a clean culture dish containing $3 \mathrm{ml}$ of $0.25 \%$ 
trypsin-EDTA and then forced through a $1-\mathrm{ml}$ syringe with a 16-gauge needle. The tissue homogenate was incubated for $30 \mathrm{~min}$ at $37^{\circ} \mathrm{C}$ in $10 \mathrm{ml}$ trypsin-EDTA solution, triturated by drawing the suspension, and then evenly divided into two or three $10-\mathrm{cm}$ tissue culture dishes in DMEM. Early-passage MEFs (passage 1-5), ATM $W T$, and $K O$ MEFs were used for all experiments, and at least three lines were examined for all studies. All media contained $10 \%$ (15\%; MEFs, IMR-90, and WI-38 cells) heat-inactivated FBS (Gibco-Invitrogen), sodium bicarbonate (2 mg/ml; SigmaAldrich, St Louis, MO, USA), penicillin (100 units $/ \mathrm{ml})$, and streptomycin $(100 \mu \mathrm{g} / \mathrm{ml}$; Gibco-Invitrogen). $N$-carbobenzoxy-lleucinyl-lleucinyl-l-norleucinal (MG132), MK 2206, roscovitine, cycloheximide (CHX), and 4-OHT were purchased from SigmaAldrich. TWS 119, ZM 447439, RO-3306, CDK1 inhibitor; SNS032 (BMS-387032), CDK1 inhibitor; PD0332991 and CDK4 inhibitor were purchased from Tocris Bioscience and BI 2536 was purchased from Selleckchem.

\section{3-D organoid assay}

For 3-D organoid assays, fibroblasts and IMR-90 cells were cultured on NanoCulture plates (SCIVAX). After seeding cells, cells were cultured in the same condition with a 2-D culture and cell images were observed and recovered 0, 3, 5, and 7 days. Cells were lysed in Spheroid Lysis Buffer (Scivax).

\section{Plasmids}

Myc-tagged WSB1 (empty and WT, $\Delta \mathrm{WD1} 1-2, \Delta \mathrm{WD} 1-3, \Delta \mathrm{N}$, $\Delta \mathrm{SOCS}, \Delta \mathrm{C}, \Delta \mathrm{FDL}$, and $\triangle \mathrm{AAA}$ ) were provided by Dr Cheol Yong Choi (Sungkyunkwan University, Korea). GFP-tagged H-Ras ${ }^{\text {V12 }}$ and c-Myc (MSCV-IRES GFP) were obtained from Addgene. GST-tagged ATM (empty and 8 overlapping GST-ATM fragments: 2-430, 400-860, 830-1 290, 1 260-1 720, 1 690-2 120, 2 0902 500, 2 400-2 700, and 2 680-3 056) were provided by Dr Titia DeLange (The Rockefeller University, New York, NY, USA) and were described earlier [43]. WSB1 and ATM mutations were generated by site-directed mutagenesis (Stratagene).

Transient transfection, viral infection, and stable transduction

shRNAs or viral vectors were infected using Lipofectamine 2000 reagent (Invitrogen). Human WSB1 and mouse WSB1 shRNAs were obtained from Sigma-Aldrich and Open Biosystems.

WSB1 shRNA (human): Open Biosystems

5'-GCTGTTGACAGTGAGCGCGGAGTTTCTCTCGTATCGTATTAGTGAAGCCACAGATGTAATACGATACGAGAGAAACTCCATGCCTACTGCCTCGGA-3'

5'-GCTGTTGACAGTGAGCGCGCTGTAAAGTGCAAGGAAATTTAGTGAAGCCACAGATGTAAATTTCCTTGCACTTTACAGCATGCCTACTGCCTCGGA-3'

WSB 1 shRNA (Mouse): Sigma-Aldrich

5'-ACATGAGCTGCTGCTATATAT-3'

5'-GCTTACTCCTTGTATCAGCTT-3'

ATM shRNA (Mouse): Sigma-Aldrich

5'-CCGTGGAGATTTCTCAATCTT-3'

5'-CCTCCAATTCTTCAGCGTAAT-3'

5'-GCTGAGACAAATAATGTCTTT-3'

5'-CGATGGAAGTTATGCGGAGTT-3'

5'-CCACCATATTTGGACAGGAAT-3'

For transient overexpression studies, DNA plasmids were transfected using Lipofectamine 2000 reagent (Invitrogen). Stable overexpression and silencing were obtained by transducing IMR90 and HEK 293T cells with retroviral or lentiviral vectors. The efficiency of knock-down or overexpression was controlled by Western blotting. Infected cells were selected with $2 \mu \mathrm{g} / \mathrm{ml}$ puromycin (Sigma-Aldrich).

\section{Colony formation and SA-B-gal}

For colony-formation or foci assay, early-passage MEFs (passage 5) cells were plated at low density into 60-mm cell culture plates. When sufficient colonies were visible, typically after 2-3 weeks, cells were washed twice in PBS before fixing in ice-cold $70 \%$ methanol for $30 \mathrm{~min}$ and then stained by $0.2 \%$ crystal violet for $0.5-1 \mathrm{~h}$. The following day, cells were rinsed in PBS and air dried. For SA- $\beta$-Gal, MEFs were fixed in $2 \%$ formaldehyde $/ 0.2 \%$ glutaraldehyde in PBS for $10 \mathrm{~min}$ and stained for SA- $\beta$-Gal according to manufacturer's instructions (Cell Signaling) overnight at $37^{\circ} \mathrm{C}$.

\section{Protein stabilization analysis}

For protein stabilization analysis, HeLa cells were transfected with the indicated constructs. After transfection for $48 \mathrm{~h}$, cells were treated with CHX $(20 \mu \mathrm{g} / \mathrm{ml})$. The cell lysates were prepared and analyzed by Western blot analysis. After CHX treatment, endogenous or exogenous Myc levels were quantified by densitometric scanning in the image J program.

\section{Co-immunoprecipitation assays, immunoblotting, and anti- bodies}

To study endogenous WSB1/ATM binding, the cells were treated with or without $10 \mu \mathrm{M}$ MG132 for $2 \mathrm{~h}$. One confluent 15$\mathrm{cm}$ dish of HEK 293T cells was lysed by sonication in NETN buffer (20 mM Tris-HCl, pH 8.0, $100 \mathrm{mM} \mathrm{NaCl}{ }_{2}, 1 \mathrm{mM}$ EDTA, and $0.5 \%$ Nonidet P-40) containing $50 \mathrm{mM}$ b-glycerophosphate, 10 $\mathrm{mM} \mathrm{NaF}$, and $1 \mathrm{mg} / \mathrm{ml}$ each of pepstatin A and aprotinin, freshly supplemented with a protease inhibitor cocktail (Roche). Prior to immunoprecipitation, protein-A-bound agarose beads were incubated overnight with ATM (Abcam, ab32420), p-ATM (Abcam, ab81292), WSB1 (Abcam, ab68953; Sigma, HPA003293; Proteintech, 1166-1-AP), Ki-67 (Abcam, ab15580), p53 (DO-1; SantaCruz, sc-126), p16 (Abcam, ab51243), and ATR antibodies in PBS with $5 \%$ BSA at $4{ }^{\circ} \mathrm{C}$. We used WSB1 antibody of ab68953 for most data (HPA003293 for IHC). We then added to the extracts before immunoprecipitation with protein-A sepharose at $4{ }^{\circ} \mathrm{C}$ for $4 \mathrm{~h}$. After three washings in a binding buffer, co-purified proteins were analyzed by Western blotting. For the mapping of the ATMinteracting domain of WSB1, HEK 293T cells were transfected with a $1 \mu \mathrm{g} / 10 \mathrm{~cm}$ dish of either wild-type or mutant forms of Myc-WSB1 and harvested in the presence of $10 \mu \mathrm{M}$ MG132. Cell extract was then subjected to immunoprecipitation using antiMyc. For removing heavy-chain, heavy- or light-chain-specific anti-mouse and anti-rabbit IgG, secondary antibodies were obtained from Jackson Immunoresearch. Anti- $\alpha$-tubulin, Myc, and HA mouse antibodies were purchased from Sigma. To study WSB1 dimerization and/or monomerizaton, reduced samples were prepared with a reducing sample buffer containing $\beta$-ME and SDS, and then boiled. Non-reducing samples were put in the sample buffer without $\beta$-ME, which were not boiled. To study WSB1 phosphorylation by CDK2, we used an anti-Phospho-(Thr) 
MAPK/CDK substrate (Cell Signaling). Antibodies used in the other studies included the following: CDK1 (Abcam, ab32384), CDK2 (Abcam, ab64669), and CDK4 (Abcam, ab137675).

\section{In vivo ubiquitination assays}

For in vivo ubiquitination, including domain mapping of WSB1, cells were transfected with ubiquitin-His plasmid together with Myc or Myc-WSB1, followed by treatment with MG 132 (10 $\mu \mathrm{M})$. Forty-eight hours post-transfection, cells were lysed by Urea lysis buffer (8 M urea, $0.1 \mathrm{M} \mathrm{Na}_{2} \mathrm{HPO}_{4}, 0.1 \mathrm{M}$ Tris/HCl (pH 8.0), $0.05 \%$ Tween 20 , and $0.01 \mathrm{M}$ imidazole). After centrifugation, the supernatants were collected and incubated with $20 \mathrm{ml} \mathrm{Ni-NTA}$ agarose beads (Qiagen) for $4 \mathrm{~h}$ at $4{ }^{\circ} \mathrm{C}$. The precipitates were washed three times with Urea wash buffer ( $8 \mathrm{M}$ urea, $0.1 \mathrm{M} \mathrm{Na}_{2} \mathrm{HPO}_{4}, 0.1$ $\mathrm{M}$ Tris $/ \mathrm{HCl}$ ( $\mathrm{pH} 8.0$ ), $0.05 \%$ Tween 20 , and $0.02 \mathrm{M}$ imidazole) and Native wash buffer $\left(0.1 \mathrm{M} \mathrm{Na}_{2} \mathrm{HPO}_{4}, 0.1 \mathrm{M}\right.$ Tris/ $\mathrm{HCl}(\mathrm{pH} 8.0)$, $0.05 \%$ Tween 20 , and $0.02 \mathrm{M}$ imidazole), boiled with SDS loading buffer, and then subjected to SDS-PAGE followed by immunoblot analysis.

\section{In vitro binding assay}

GST fusion proteins were prepared following standard protocol [79]. For in vitro biding assays, ATM (8 deleted mutants) GST fusion proteins bound to the GSH Sepharose were incubated with cell lysates. After washing, the bound proteins were separated by SDS-PAGE and immunoblotted with indicated antibodies.

\section{Immunofluorescence}

For immunofluorescence staining, MEF and IMR-90 cells were plated on glass cover slips and infected with the indicated constructs. Cells were then fixed in $3.7 \%$ paraformaldehyde for $10 \mathrm{~min}$ at room temperature and stained using standard protocols. Immunofluorescence images were taken using fluorescent microscopy (Nikon Microscope, Melville, NY, USA).

\section{Proliferation and viability analysis}

For proliferation staining, MEFs were plated on glass cover slips and infected with the indicated constructs. Cells were then fixed and stained with Ki-67 antibody. For viability staining, MEF cells were infected with the indicated constructs and were seeded in six-well plates and cultured for 15 days. Cells were then washed three times with PBS and stained with $0.2 \%$ crystal violet for 30 min before cell counting under microscopy.

\section{In vitro kinase assay}

HEK 293T cells were transfected with Flag-CDK2 and V5-CyclinE. Forty-eight hours later, the cells were collected and the cell lysates were subjected to immunoprecipitated anti-Flag antibody. The protein conjugated to the beads were eluted with Flag peptides and subjected to in vitro kinase assay, or the active CDK2/CyclinE protein from Millipore was used as kinase in the assay. GST and GST-WSB1 expressed in bacteria were purified and subjected to kinase assay as substrates. The phosphorylation of WSB1 by CDK2 was examined using an anti-CDK substrate antibody.

\section{Reverse transcription-PCR of $c D N A$}

RNA preparation and cDNA were described previously [80]. The following primers were used:

$$
\text { WSB } 1 \text { forward 5'-TCTCCTGACTCTTCTATGCTGTGT-3' }
$$

reverse 5'-CATGGTGTATTTATCCATATTCCAAA-3' ATM forward 5'-GTGTTCTGAAATTGTGAACCATGAGTCT AGT-3'

reverse 5'-TGGTATCTTCATTAAAAACCTGGTGACAGA-3' forward 5'-GATGGAGAAAGTAGTGATGAGC-3' reverse 5'-AGTCACCAGATTTCCATATTCTC-3'

The $\beta$-actin sequence was described previously [80].

\section{Electroporation}

For complementation experiments, Vector, Flag-ATM-WT, or Flag-ATM mutant plasmids (K1109R, K1114R, K1323R, K1572R, K2025R, and 2KR), Flag-CDK1, Flag-CDK2, Flag-CDK4, or HA-WSB1 were electroporated into ATM KO MEFs using GenePulser Xcell (Bio-Rad). Pellet cells were resuspended in $10 \mathrm{ml}$ of DMEM. The cells were either re-suspended $\left(10^{7}\right)$ in $400 \mu \mathrm{l}$ of transfection serum-free medium and $10-40 \mu \mathrm{g}$ of plasmid DNA added or re-suspended to the cells $\left(5 \times 10^{7}\right)$ in $400 \mu 1$ of transfection serum-free medium and $40 \mu \mathrm{g}$ of plasmid DNA added. Electroporation used the following settings: square wave pulse, $200 \mathrm{~V}$, $30 \mathrm{~ms}$, and $4 \mathrm{~mm}$ cuvette. The cuvette was placed on ice for 5-10 min. Cells were transferred to a $60-\mathrm{mm}$ dish and $4 \mathrm{ml}$ of media added ( $15 \%$ serum; without antibiotics and selective agent). Cells were then incubated overnight and media was changed at the next day.

\section{Mouse xenograft tumor model}

For MEF xenograft experiments, cells were infected (or transfected) with the indicated constructs (by electroporation) and injected into nude mice. Equal numbers $\left(1 \times 10^{6}\right)$ of infected MEF cells expressing luciferase mixed at a 1:1 dilution with Matrigel (Collaborative Research) were implanted in the backs of athymic nude mice. For CDK-related xenograft experiments, mice were treated with CDKIs, such as RO-3306 (CDK1 inhibitor), SNS-032 (BMS-387032, CDK1 inhibitor) and PD0332991 (CDK4 inhibitor). Tumor growth was monitored using calipers and visualized with a bioluminescence-based IVIS system (Caliper LifeScience). Tumor growth was measured using a Vernier caliper at the indicated times after injection, and the tumor volume was calculated as length $\times$ width $\times$ height. Tumor size was monitored by measuring mice two times a week. When tumors reached $2 \mathrm{~cm}$ in diameter, mice were euthanized.

\section{Immunohistochemistry}

All of step for IHC were prepared following standard protocol. Briefly, immunohistochemical cytokeratin staining was performed on formalin-fixed, paraffin-embedded tissue using an indirect immunoperoxidase technique. Sections mounted on silanized slides were dewaxed in xylene, dehydrated in ethanol, boiled in $0.01 \mathrm{M}$ citrate buffer ( $\mathrm{pH} \mathrm{6.0)}$ for $20 \mathrm{~min}$ in a microwave oven and then incubated with $3 \%$ hydrogen peroxide for $5 \mathrm{~min}$. After washing with PBS, the slides were incubated in $10 \%$ normal BSA for 5 min, followed by incubation for 45 min with monoclonal mouse anti-human WSB1 (1:200, Sigma-Aldrich) and anti-ATM (1:100, Abcam). After washing, sections were incubated with labeled polymer (Bond Polymer Refine Detection) and diaminobenzidine. The sections were then counterstained with hematoxylin, dehydrated, cleared, and mounted.

\section{Statistical analysis}


Each assay was performed in triplicate and independently repeated at least three times. The results are presented as mean \pm SEM. Statistical analyses were performed using GraphPad Prism software (version 4.02; GraphPad Software, San Diego, CA, USA). One-way analysis of variance followed by $t$-testing was used to compare the results. A difference was considered significant if $P<0.05$. Statistical significance was defined as $P<0.05\left(^{*}\right)$, $P<0.01$ (**) $^{*} P<0.001$ (***), and $P<0.0001$ (****).

\section{Acknowledgments}

We thank all members of Dr Lou's laboratory (Dr K Luo, Dr J Yuan, Dr H Pei, Dr B Qin, Dr T Liu, Dr H Zhang, Dr M Deng, Dr Y Li, Dr F Yuan, Dr W Guo, Dr L Zhang, Dr B Xu, Dr H Shi, S Nowsheen, and Y-H Lin) for their critical discussions. We thank Dr MS Kim and Dr CH Park for performing the animal experiment. We thank Dr CY Choi for WSB1 plasmids and Dr Y Ikeda for luciferase lentivirus plasmid. We thank Dr Kah Whye Peng and Diana L Escobar for bioluminescence-based IVIS system. We also thank Dr Z Zhang and JS Lee for ER-RAS-IMR-90 cells, and Dr Titia DeLange (The Rockefeller University) and Dr Hui-Kuan Lin (Wake Forest School of Medicine) for GST-ATM plasmids. This work was supported by 2015DFA30610, NIH grants CA130996, CA203561, and CA189666 to ZL.

\section{Author Contributions}

SBL, JJK, and ZL designed and performed most of the experiments, analyzed data, and prepared the manuscript as lead authors. SYY, SHK, SAH, JML, and SYT provided clinical samples and analyzed data. PY and BG performed animal experiments. ZL designed and supervised the study.

\section{Competing Financial Interests}

The authors declare no competing financial interests.

\section{References}

1 Lowe SW, Cepero E, Evan G. Intrinsic tumour suppression. Nature 2004; 432:307-315.

2 Collado M, Gil J, Efeyan A, et al. Tumour biology: senescence in premalignant tumours. Nature 2005; 436:642.

3 Munoz-Espin D, Serrano M. Cellular senescence: from physiology to pathology. Nat Rew Mol Cell Biol 2014; 15:482-496.

$4 \mathrm{Xu} \mathrm{Y,} \mathrm{Li} \mathrm{N,} \mathrm{Xiang} \mathrm{R,} \mathrm{Sun} \mathrm{P.} \mathrm{Emerging} \mathrm{roles} \mathrm{of} \mathrm{the} \mathrm{p38}$ MAPK and PI3K/AKT/mTOR pathways in oncogene-induced senescence. Trends Biochem Sci 2014; 39:268-276.

5 Halazonetis TD, Gorgoulis VG, Bartek J. An oncogene-induced DNA damage model for cancer development. Science 2008; 319:1352-1355.

6 Venkitaraman AR. Medicine: aborting the birth of cancer. $\mathrm{Na}$ ture 2005; 434:829-830.

7 Bartek J, Bartkova J, Lukas J. DNA damage signalling guards against activated oncogenes and tumour progression. Oncogene 2007; 26:7773-7779.

8 Hills SA, Diffley JF. DNA replication and oncogene-induced replicative stress. Curr Biol 2014; 24:R435-R444.

9 Reddy JP, Peddibhotla S, Bu W, et al. Defining the ATM-mediated barrier to tumorigenesis in somatic mammary cells following ErbB2 activation. Proc Natl Acad Sci USA 2010; 107:3728-3733.

10 Sulli G, Di Micco R, d'Adda di Fagagna F. Crosstalk between chromatin state and DNA damage response in cellular senescence and cancer. Nat Rev Cancer 2012; 12:709-720.

11 Aird KM, Worth AJ, Snyder NW, et al. ATM couples replication stress and metabolic reprogramming during cellular senescence. Cell Rep 2015; 11:893-901.

12 Bartkova J, Horejsi Z, Koed K, et al. DNA damage response as a candidate anti-cancer barrier in early human tumorigenesis. Nature 2005; 434:864-870.

13 Campisi J. Cellular senescence: putting the paradoxes in perspective. Curr Opin Genet Dev 2011; 21:107-112.

14 Gorgoulis VG, Vassiliou LV, Karakaidos P, et al. Activation of the DNA damage checkpoint and genomic instability in human precancerous lesions. Nature 2005; 434:907-913.

15 Reimann M, Loddenkemper C, Rudolph C, et al. The Mycevoked DNA damage response accounts for treatment resistance in primary lymphomas in vivo. Blood 2007; 110:29963004.

16 Pusapati RV, Rounbehler RJ, Hong S, et al. ATM promotes apoptosis and suppresses tumorigenesis in response to Myc. Proc Natl Acad Sci USA 2006; 103:1446-1451.

17 Bartkova J, Rezaei N, Liontos M, et al. Oncogene-induced senescence is part of the tumorigenesis barrier imposed by DNA damage checkpoints. Nature 2006; 444:633-637.

18 Di Micco R, Fumagalli M, Cicalese A, et al. Oncogene-induced senescence is a DNA damage response triggered by DNA hyper-replication. Nature 2006; 444:638-642.

19 Mallette FA, Gaumont-Leclerc MF, Ferbeyre G. The DNA damage signaling pathway is a critical mediator of oncogene-induced senescence. Genes Dev 2007; 21:43-48.

20 Aird KM, Zhang R. ATM in senescence. Oncotarget 2015; 6:14729-14730.

21 Suram A, Kaplunov J, Patel PL, et al. Oncogene-induced telomere dysfunction enforces cellular senescence in human cancer precursor lesions. EMBO J 2012; 31:2839-2851.

22 Shiloh Y, Ziv Y. The ATM protein kinase: regulating the cellular response to genotoxic stress, and more. Nat Rev Mol Cell Biol 2013; 14:197-210.

23 Kastan MB, Lim DS. The many substrates and functions of ATM. Nat Rev Mol Cell Biol 2000; 1:179-186.

24 Silins I, Finnberg N, Stahl A, Hogberg J, Stenius U. Reduced ATM kinase activity and an attenuated p53 response to DNA damage in carcinogen-induced preneoplastic hepatic lesions in the rat. Carcinogenesis 2001; 22:2023-2031.

25 Angele S, Jones C, Reis Filho JS, et al. Expression of ATM, $\mathrm{p} 53$, and the MRE11-Rad50-NBS1 complex in myoepithelial cells from benign and malignant proliferations of the breast. $J$ Clin Pathol 2004; 57:1179-1184.

26 Feng X, Li H, Dean M, et al. Low ATM protein expression in malignant tumor as well as cancer-associated stroma are independent prognostic factors in a retrospective study of early-stage hormone-negative breast cancer. Breast Cancer Res 2015; 17:65.

27 Choi DW, Seo YM, Kim EA, et al. Ubiquitination and degradation of homeodomain-interacting protein kinase 2 by WD40 repeat/SOCS box protein WSB-1. J Biol Chem 2008; 283:4682-4689. 
28 Vasiliauskas D, Hancock S, Stern CD. SWiP-1: novel SOCS box containing WD-protein regulated by signalling centres and by Shh during development. Mech Dev 1999; 82:79-94.

29 Rhodes DR, Chinnaiyan AM. Integrative analysis of the cancer transcriptome. Nat Genet 2005; 37 Suppl:S31-37.

30 Benita Y, Kikuchi H, Smith AD, Zhang MQ, Chung DC, Xavier RJ. An integrative genomics approach identifies Hypoxia Inducible Factor-1 (HIF-1)-target genes that form the core response to hypoxia. Nucleic Acids Res 2009; 37:45874602.

31 Archange C, Nowak J, Garcia S, et al. The WSB1 gene is involved in pancreatic cancer progression. PloS One 2008; 3:e2475.

32 Silva AS, Wood SH, van Dam S, Berres S, McArdle A, de Magalhaes JP. Gathering insights on disease etiology from gene expression profiles of healthy tissues. Bioinformatics 2011; 27:3300-3305.

33 Tong Y, Li QG, Xing TY, Zhang M, Zhang JJ, Xia Q. HIF1 regulates WSB-1 expression to promote hypoxia-induced chemoresistance in hepatocellular carcinoma cells. FEBS Lett 2013; 587:2530-2535.

34 Kim JJ, Lee SB, Jang J, et al. WSB1 promotes tumor metastasis by inducing pVHL degradation. Genes Dev 2015; 29:2244-2257.

35 Evangelou K, Bartkova J, Kotsinas A, et al. The DNA damage checkpoint precedes activation of ARF in response to escalating oncogenic stress during tumorigenesis. Cell Death Differ 2013; 20:1485-1497.

36 Pampaloni F, Reynaud EG, Stelzer EH. The third dimension bridges the gap between cell culture and live tissue. Nat Rev Mol Cell Biol 2007; 8:839-845.

37 Jechlinger M. Organotypic culture of untransformed and tumorigenic primary mammary epithelial cells. Cold Spring Harb Protoc 2015; 2015:pdb prot078295.

38 Di Micco R, Sulli G, Dobreva M, et al. Interplay between oncogene-induced DNA damage response and heterochromatin in senescence and cancer. Nat Cell Biol 2011; 13:292-302.

39 Luo X, Suzuki M, Ghandhi SA, Amundson SA, Boothman DA. ATM regulates insulin-like growth factor 1-secretory clusterin (IGF-1-sCLU) expression that protects cells against senescence. PloS One 2014; 9:e99983.

40 Abdel-Fatah TM, Arora A, Alsubhi N, et al. Clinicopathological significance of ATM-Chk2 expression in sporadic breast cancers: a comprehensive analysis in large cohorts. Neoplasia 2014; 16:982-991.

41 Bueno RC, Canevari RA, Villacis RA, et al. ATM down-regulation is associated with poor prognosis in sporadic breast carcinomas. Ann Oncol 2014; 25:69-75.

42 Dentice M, Bandyopadhyay A, Gereben B, et al. The Hedgehog-inducible ubiquitin ligase subunit WSB-1 modulates thyroid hormone activation and PTHrP secretion in the developing growth plate. Nat Cell Biol 2005; 7:698-705.

43 Takai H, Wang RC, Takai KK, Yang H, de Lange T. Tel2 regulates the stability of PI3K-related protein kinases. Cell 2007; 131:1248-1259.

$44 \mathrm{Xu} \mathrm{C}$, Min J. Structure and function of WD40 domain proteins. Protein Cell 2011; 2:202-214.

45 Zhang JG, Farley A, Nicholson SE, et al. The conserved SOCS box motif in suppressors of cytokine signaling binds to elongins B and C and may couple bound proteins to proteasomal degradation. Proc Natl Acad Sci USA 1999; 96:20712076.

46 Pozzebon ME, Varadaraj A, Mattoscio D, et al. BC-box protein domain-related mechanism for VHL protein degradation. Proc Natl Acad Sci USA 2013; 110:18168-18173.

47 Coppe JP, Desprez PY, Krtolica A, Campisi J. The senescence-associated secretory phenotype: the dark side of tumor suppression. Annu Rev Pathol 2010; 5:99-118.

48 Yin Q, Lin SC, Lamothe B, et al. E2 interaction and dimerization in the crystal structure of TRAF6. Nat Struct Mol Biol 2009; 16:658-666.

49 Liu T, Zhang H, Xiong J, Yi S, Gu L, Zhou M. Inhibition of MDM2 homodimerization by XIAP IRES stabilizes MDM2, influencing cancer cell survival. Mol Cancer 2015; 14:65.

50 de Bie P, Ciechanover A. Ubiquitination of E3 ligases: self-regulation of the ubiquitin system via proteolytic and non-proteolytic mechanisms. Cell Death Differ 2011; 18:1393-1402.

51 Xue Y, Ren J, Gao X, Jin C, Wen L, Yao X. GPS 2.0, a tool to predict kinase-specific phosphorylation sites in hierarchy. Mol Cell Proteomics 2008; 7:1598-1608.

52 Campaner S, Doni M, Hydbring P, et al. Cdk2 suppresses cellular senescence induced by the c-myc oncogene. Nat Cell Biol 2010; 12:54-59.

53 van Riggelen J, Felsher DW. Myc and a Cdk2 senescence switch. Nat Cell Biol 2010; 12:7-9.

54 Lents NH, Keenan SM, Bellone C, Baldassare JJ. Stimulation of the Raf/MEK/ERK cascade is necessary and sufficient for activation and Thr-160 phosphorylation of a nuclear-targeted CDK2. J Biol Chem 2002; 277:47469-47475.

55 Chambard JC, Lefloch R, Pouyssegur J, Lenormand P. ERK implication in cell cycle regulation. Biochim Biophys Acta 2007; 1773:1299-1310.

56 Gysin S, Lee SH, Dean NM, McMahon M. Pharmacologic inhibition of $\mathrm{RAF} \rightarrow \mathrm{MEK} \rightarrow \mathrm{ERK}$ signaling elicits pancreatic cancer cell cycle arrest through induced expression of p27Kip1. Cancer Res 2005; 65:4870-4880.

57 Meloche S, Pouyssegur J. The ERK1/2 mitogen-activated protein kinase pathway as a master regulator of the G1- to S-phase transition. Oncogene 2007; 26:3227-3239.

58 Yu Q, Geng Y, Sicinski P. Specific protection against breast cancers by cyclin D1 ablation. Nature 2001; 411:1017-1021.

59 Dulic V, Drullinger LF, Lees E, Reed SI, Stein GH. Altered regulation of G1 cyclins in senescent human diploid fibroblasts: accumulation of inactive cyclin E-Cdk2 and cyclin D1Cdk2 complexes. Proc Natl Acad Sci USA 1993; 90:1103411038.

60 Stein GH, Drullinger LF, Soulard A, Dulic V. Differential roles for cyclin-dependent kinase inhibitors p21 and p16 in the mechanisms of senescence and differentiation in human fibroblasts. Mol Cell Biol 1999; 19:2109-2117.

61 McConnell BB, Starborg M, Brookes S, Peters G. Inhibitors of cyclin-dependent kinases induce features of replicative senescence in early passage human diploid fibroblasts. Curr Biol 1998; 8:351-354.

62 Kong Y, Cui H, Ramkumar C, Zhang H. Regulation of senescence in cancer and aging. J Aging Res 2011; 2011:963172.

63 Cao J, Wang Y, Dong R, et al. Hypoxia-induced WSB1 pro- 
motes the metastatic potential of osteosarcoma cells. Cancer Res 2015; 75:4839-4851.

64 Kim HS, Kim MA, Hodgson D, et al. Concordance of ATM (ataxia telangiectasia mutated) immunohistochemistry between biopsy or metastatic tumor samples and primary tumors in gastric cancer patients. Pathobiology 2013; 80:127-137.

65 Angele S, Falconer A, Edwards SM, et al. ATM polymorphisms as risk factors for prostate cancer development. $\mathrm{Br} J$ Cancer 2004; 91:783-787.

$66 \mathrm{Kim} \mathrm{JH}, \mathrm{Kim} \mathrm{H}$, Lee KY, et al. Genetic polymorphisms of ataxia telangiectasia mutated affect lung cancer risk. Hum Mol Genet 2006; 15:1181-1186.

67 Stredrick DL, Garcia-Closas M, Pineda MA, et al. The ATM missense mutation p.Ser49Cys (c.146C $>$ G) and the risk of breast cancer. Hum Mutat 2006; 27:538-544.

68 Hall J. The Ataxia-telangiectasia mutated gene and breast cancer: gene expression profiles and sequence variants. Cancer Lett 2005; 227:105-114.

69 Angele S, Treilleux I, Bremond A, Taniere P, Hall J. Altered expression of DNA double-strand break detection and repair proteins in breast carcinomas. Histopathology 2003; 43:347353.

70 Grabsch H, Dattani M, Barker L, et al. Expression of DNA double-strand break repair proteins ATM and BRCA1 predicts survival in colorectal cancer. Clin Cancer Res 2006; 12:14941500 .

71 Kang B, Guo RF, Tan XH, Zhao M, Tang ZB, Lu YY. Expression status of ataxia-telangiectasia-mutated gene correlated with prognosis in advanced gastric cancer. Mutat Res 2008; 638: 17-25.

72 Tribius S, Pidel A, Casper D. ATM protein expression cor- relates with radioresistance in primary glioblastoma cells in culture. Int J Radiat Oncol Biol Phys 2001; 50:511-523.

73 Hsia SM, Yu CC, Shih YH, et al. Isoliquiritigenin as a cause of DNA damage and inhibitor of ataxia-telangiectasia mutated expression leading to $\mathrm{G} 2 / \mathrm{M}$ phase arrest and apoptosis in oral squamous cell carcinoma. Head Neck 2016; 38 Suppl 1:E360-E371.

74 Flanagan JM, Munoz-Alegre M, Henderson S, et al. Genebody hypermethylation of ATM in peripheral blood DNA of bilateral breast cancer patients. Hum Mol Genet 2009; 18:1332-1342.

75 Vo QN, Kim WJ, Cvitanovic L, Boudreau DA, Ginzinger DG, Brown KD. The ATM gene is a target for epigenetic silencing in locally advanced breast cancer. Oncogene 2004; 23:94329437.

76 Kairouz R, Clarke RA, Marr PJ, et al. ATM protein synthesis patterns in sporadic breast cancer. Mol Pathol 1999; 52:252256.

77 Fang Z, Kozlov S, McKay MJ, et al. Low levels of ATM in breast cancer patients with clinical radiosensitivity. Genome Integr 2010; 1:9.

78 Dairkee SH, Ji Y, Ben Y, Moore DH, Meng Z, Jeffrey SS. A molecular 'signature' of primary breast cancer cultures; patterns resembling tumor tissue. BMC Genomics 2004; 5:47.

79 Lee SB, Kim JJ, Nam HJ, et al. Parkin regulates mitosis and genomic stability through Cdc20/Cdh1. Mol Cell 2015; 60:2134.

80 Lee SB, Kim JJ, Chung JS, et al. Romo1 is a negative-feedback regulator of Myc. J Cell Sci 2011; 124:1911-1924.

(Supplementary information is linked to the online version of the paper on the Cell Research website) 